\title{
Least-energy nodal solutions of critical Kirchhoff problems with logarithmic nonlinearity
}

\author{
Sihua Liang ${ }^{1,2} \cdot$ Vicenţiu D. Rădulescu ${ }^{3,4}$
}

Received: 2 July 2020 / Accepted: 20 August 2020 / Published online: 9 September 2020

(c) The Author(s) 2020

\section{Abstract}

In this paper, we are concerned with the existence of least energy sign-changing solutions for the following fractional Kirchhoff problem with logarithmic and critical nonlinearity:

$$
\begin{cases}\left(a+b[u]_{s, p}^{p}\right)(-\Delta)_{p}^{s} u=\lambda|u|^{q-2} u \ln |u|^{2}+|u|_{s}^{p_{s}^{*}-2} u & \text { in } \Omega, \\ u=0 & \text { in } \mathbb{R}^{N} \backslash \Omega\end{cases}
$$

where $N>s p$ with $s \in(0,1), p>1$, and

$$
[u]_{s, p}^{p}=\iint_{\mathbb{R}^{2 N}} \frac{|u(x)-u(y)|^{p}}{|x-y|^{N+p s}} d x d y,
$$

$p_{s}^{*}=N p /(N-p s)$ is the fractional critical Sobolev exponent, $\Omega \subset \mathbb{R}^{N}(N \geq 3)$ is a bounded domain with Lipschitz boundary and $\lambda$ is a positive parameter. By using constraint variational methods, topological degree theory and quantitative deformation arguments, we prove that the above problem has one least energy sign-changing solution $u_{b}$. Moreover, for any $\lambda>0$, we show that the energy of $u_{b}$ is strictly larger than two times the ground state energy. Finally, we consider $b$ as a parameter and study the convergence property of the least energy sign-changing solution as $b \rightarrow 0$.

Keywords Fractional $p$-Laplacian · Critical problem · Logarithmic nonlinearity . Variational methods · Nodal solution

Mathematics Subject Classification 35A15 · 35J60 - 47G20

Vicenţiu D. Rădulescu radulescu@inf.ucv.ro

Extended author information available on the last page of the article 


\section{Introduction}

In this paper, we are interested in the existence, energy estimates and the convergence property of the least energy sign-changing solution for the following fractional Kirchhoff problems with logarithmic and critical nonlinearity:

$$
\begin{cases}\left(a+b[u]_{s, p}^{p}\right)(-\Delta)_{p}^{s} u=\lambda|u|^{q-2} u \ln |u|^{2}+|u|^{p_{s}^{*}-2} u & \text { in } \Omega, \\ u=0 & \text { in } \mathbb{R}^{N} \backslash \Omega,\end{cases}
$$

where $N>s p$ with $s \in(0,1), p>1$, and

$$
[u]_{s, p}^{p}=\iint_{\mathbb{R}^{2 N}} \frac{|u(x)-u(y)|^{p}}{|x-y|^{N+p s}} d x d y,
$$

$p_{s}^{*}=N p /(N-p s)$ is the fractional critical Sobolev exponent, $\Omega \subset \mathbb{R}^{N}(N \geq 3)$ is a bounded domain with Lipshcitz boundary and $\lambda$ is a positive parameter. We denote by $(-\Delta)_{p}^{s}$ the fractional $p$-Laplace operator which, up to a normalization constant, is defined as

$$
(-\Delta)_{p}^{s} \varphi(x)=2 \lim _{\varepsilon \rightarrow 0^{+}} \int_{\mathbb{R}^{N} \backslash B_{\varepsilon}(x)} \frac{|\varphi(x)-\varphi(y)|^{p-2}(\varphi(x)-\varphi(y))}{|x-y|^{N+p s}} d y, \quad x \in \mathbb{R}^{N},
$$

for all $\varphi \in C_{0}^{\infty}\left(\mathbb{R}^{N}\right)$. Henceforward, $B_{\varepsilon}(x)$ denotes the open ball of $\mathbb{R}^{N}$ centered at $x \in \mathbb{R}^{N}$ and radius $\varepsilon>0$.

One of the classical topics in the qualitative analysis of PDEs is the study of existence and multiplicity properties of solutions for both the Kirchhoff problems and the fractional Kirchhoff problems under various hypotheses on the nonlinearity. In the recent past there is a vast literature concerning the existence and multiplicity of solutions for the following Dirichlet problem of Kirchhoff type

$$
\left\{\begin{array}{l}
-\left(a+b \int_{\Omega}|\nabla u|^{2} d x\right) \Delta u=f(x, u), \quad x \in \Omega \\
\left.u\right|_{\partial \Omega}=0
\end{array}\right.
$$

Problem (1.2) is a generalization of a model introduced by Kirchhoff [24]. More precisely, Kirchhoff proposed a model given by the equation

$$
\rho \frac{\partial^{2} u}{\partial t^{2}}-\left(\frac{\rho_{0}}{h}+\frac{E}{2 L} \int_{0}^{L}\left|\frac{\partial u}{\partial x}\right|^{2} d x\right) \frac{\partial^{2} u}{\partial x^{2}}=0,
$$

where $\rho, \rho_{0}, h, E, L$ are constants. This nonlocal model extends the classical D'Alembert's wave equation, by considering the effects of the changes in the length of the strings during the vibrations. Since Lions [29] introduced an abstract framework to Kirchhoff-type equations, the solvability of these nonlocal problems has been well studied in the general dimension by various authors. We refer to D'Ancona and 
Shibata [13] and D'Ancona and Spagnolo [14] for the global solvability of various classes of Kirchhoff-type problems. We also refer to Carrier $[9,10]$ who used a more rigorous method to model transverse vibration via the coupled governing equation of planar vibration in order to recover the nonlinear integro partial-differential equation, in which a more general Kirchhoff function was considered. In addition, the nonlocal Kirchhoff problems of parabolic type can model several biological systems, such as population density, see for example Ghergu and Rădulescu [21]. For more details on mathematical theories and its applications of Kirchhoff-type problems, we refer the readers to $[4,14,23,26,27,41]$.

Problem (1.2) is a nonlocal problem because the term $b \int_{\Omega}|\nabla u|^{2} d x \Delta u$ appears in the left-hand side of the equation, which results that (1.2) is not a pointwise identity. Moreover, the energy functional associated to (1.2) has different properties with respect to the local case corresponding to $b=0$, hence several mathematical difficulties are brought naturally out in the study of the nonlocal problems $(b \neq 0)$ by means of variational methods. Recently, Fiscella and Valdinoci [19] proposed a steady-state Kirchhoff model involving the fractional Laplacian by taking into account the nonlocal aspect of the tension arising from nonlocal measurements of the fractional length of the string, see [19, Appendix A] for more details. Fractional Kirchhoff-type Laplacian problems have been studied by many authors, refer [2,3,20,28,34,36,37,48,49,51]. Here we can refer the recent monograph about nonlocal fractional problems [35]. We note that the results dealing with the problem (1.2) with critical nonlinearity are relatively scarce. The main difficulty in the study of these problems is due to the lack of compactness caused by the presence of the critical Sobolev exponent.

Recently, most of the literature deals with fractional Laplacian problems with power type nonlinearities, there are a few papers that deal with the existence and multiplicity of solutions for fractional problems involving logarithmic nonlinearity. In [15], d'Avenia et al. considered the following fractional logarithmic Schorödinger equation

$$
(-\Delta)^{s} u+\omega u=u \log |u|^{2}, \quad x \in \mathbb{R}^{N},
$$

where $\omega>0$. By employing the fractional logarithmic Sobolev inequality, [15] obtained the existence of infinitely many solutions. Moreover, the regularity of solutions was also discussed in [15]. In [42], Truong studied the following problem fractional $p$-Laplacian equations with logarithmic nonlinearity

$$
(-\Delta)_{p}^{s} u+V(x)|u|^{p-2} u=\lambda a(x)|u|^{p-2} u \ln |u|, \quad x \in \mathbb{R}^{N},
$$

where $a$ is a sign-changing function. Under some assumptions on $V, a$ and $\lambda$, [42] obtained two nontrivial solutions by using Nehari manifold approach. Very recently, Xiang, Hu and Yang in [47] considered the following Kirchhoff problems with combined nonlinearity of logarithmic and power type

$$
\begin{cases}M\left([u]_{s, p}^{p}\right)(-\Delta)_{p}^{s} u=h(x)|u|^{\theta p-2} u \ln |u|+\lambda|u|^{q-2} u & x \in \Omega, \\ u=0 & x \in \mathbb{R}^{N} \backslash \Omega,\end{cases}
$$


where $s \in(0,1), 1<p<N / s, \Omega$ is a bounded domain in $\mathbb{R}^{N}$ with Lipschitz boundary, $M\left([u]_{s, p}^{p}\right)=[u]_{s, p}^{(\theta-1) p}$ with $\theta \geq 1$, and $h$ is a sign-changing function. When $\lambda$ is sufficiently small, [47] obtained two nonnegative local least energy solutions by using Nehari manifold approach.

On the other hand, the existence of sign-changing solution of nonlinear elliptic PDEs with power nonlinearities has been studied extensively for the $p$-Laplacian operator as well as the fractional $p$-Laplacian operator. We refer the reader to see $[5,6,11,12,30,44]$ and the references therein. Consider the nonlocal problem

$$
\left\{\begin{array}{l}
(-\Delta)_{p}^{s} u=f(x, u), \quad x \in \Omega, \\
u=0, \quad x \in \mathbb{R}^{N} \backslash \Omega .
\end{array}\right.
$$

For $p=2$, the authors in [11], have studied the problem (1.4), where the fractional Laplacian operator is defined through spectral decomposition to obtain the sign-changing solution. The method of harmonic extension was introduced by Caffarelli and Silvestre [8] to transform the nonlocal problem in $\Omega$ to a local problem in the half cylinder $\Omega \times(0, \infty)$, by using an equivalent definition of the fraction Laplacian operator [7]. For $p \in(1, \infty)$, the problem studied by Chang et al. [12], where the authors have guaranteed the existence of a sign-changing solutions by using Nehari manifold method.

Recently, many authors pay their attention to find sign-changing solutions to problem (1.2) or similar Kirchhoff-type equations, and indeed, some interesting results were obtained. For example, Zhang and Perera [50] and Mao and Zhang [32] used the method of invariant sets of descent flow to obtain the existence of a sign-changing solution of problem (1.2). In [17], Figueiredo and Nascimento considered the following Kirchhoff equation of the type:

$$
\left\{\begin{array}{l}
-M\left(\int_{\Omega}|\nabla u|^{2} d x\right) \Delta u=g(u), \quad x \in \Omega \\
\left.u\right|_{\partial \Omega}=0
\end{array}\right.
$$

where $\Omega$ is a bounded domain in $\mathbb{R}^{3}, M$ is a general $C^{1}$ class function, and $g$ is a superlinear $C^{1}$ class function with subcritical growth. By using the minimization argument and a quantitative deformation lemma, the existence of a sign-changing solution for this Kirchhoff equation was obtained. In unbounded domains, Figueiredo and Santos Júnior [18] studied a class of nonlocal Schrödinger-Kirchhof problems involving only continuous functions. Using a minimization argument and a quantitative deformation lemma, they obtained a least energy sign-changing solution to Schrödinger-Kirchhof problems. Moreover, when the problem presents symmetry, the authors showed that it has infinitely many nontrivial solutions.

It is noted that combining constraint variational methods and quantitative deformation lemma, Shuai [38] proved that problem (1.2) has one least energy sign-changing solution $u_{b}$ and the energy of $u_{b}$ strictly larger than the ground state energy. Moreover, the author investigated the asymptotic behavior of $u_{b}$ as the parameter $b \searrow 0$. Later, under some more weak assumptions on $g$ (especially, Nehari type monotonicity condition been removed), with the aid of some new analytical skills and 
Non-Nehari manifold method, Tang and Cheng [40] improved and generalized some results obtained in [38].

In [16], Deng, Peng, and Shuai studied the following Kirchhoff problem:

$$
-\left(a+b \int_{\mathbb{R}^{3}}|\nabla u|^{2} d x\right) \Delta u=f(x, u), \quad x \in \mathbb{R}^{3} .
$$

The authors obtained the existence of radial sign-changing solutions with prescribed numbers of nodal domains for Kirchhoff problem (1.6) in $H_{r}^{1}\left(\mathbb{R}^{3}\right)$, the subspace of radial functions of $H^{1}\left(\mathbb{R}^{3}\right)$ by using a Nehari manifold and gluing solution pieces together, when $V(x)=V(|x|), f(x, u)=f(|x|, u)$ and satisfies some conditions. Precisely, they proved the existence of a sign-changing solution, which changes signs exactly $k$ times for any $k \in \mathbb{N}$. Moreover, they investigated the energy property and the asymptotic behavior of the sign-changing solution. By using a combination of the invariant set method and the Ljusternik-Schnirelman type minimax method, Sun et al. [39] obtained infinitely many sign-changing solutions for Kirchhoff problem (1.6) when $f(x, u)=f(u)$ and $f$ is odd in $u$. It is worth noticing that, in [39], the nonlinear term may not be 4-superlinear at infinity; in particular, it includes the power-type nonlinearity $|u|^{p-2} u$ with $p \in(2,4]$. In [43], the authors obtained the existence of least energy sign-changing solutions of Kirchhoff-type equation with critical growth by using the constraint variational method and the quantitative deformation lemma. For more results on sign-changing solutions for Kirchhoff-type equations, we refer the reader to $[16,25,31]$ and the references therein.

\section{Abstract setting and main results}

To the best of our knowledge, there are no results concerning the existence of signchanging solutions for fractional Kirchhoff problems with logarithmic and critical nonlinearity. Hence, a natural question is whether or not there exist nodal solutions of problem (1.1)? The goal of the present paper is to develop a thorough qualitative analysis in this direction.

We first recall some preliminary results on the fractional Sobolev space $W_{0}^{s, p}(\Omega)$ with respect to the norm $\|u\|=[u]_{s, p}$. We then have that $W_{0}^{s, p}(\Omega)$ is continuously and compactly embedded into the Lebesgue space $L^{r}(\Omega)$ endowed the norm $|u|_{r}=$ $\left(\int_{\Omega}|u|^{r} d x\right)^{\frac{1}{r}}, p<r<p_{s}^{*}$. Denote by $S_{r}$ the best constant for this embedding, that is,

$$
S_{r}|u|_{r} \leq\|u\|, \quad \forall u \in W_{0}^{s, p}(\Omega) .
$$

In particular, if $S$ is the the best constant for the embedding $W_{0}^{s, p}(\Omega) \hookrightarrow L^{p_{s}^{*}}(\Omega)$, then it is defined by

$$
S=\inf _{u \in W_{0}^{s, p}(\Omega) \backslash\{0\}} \frac{\iint_{\mathbb{R}^{2 N}} \frac{|u(x)-u(y)|^{p}}{|x-y|^{N+p s}} d x d y}{\left(\int_{\Omega}|u|^{p_{s}^{*}} d x\right)^{\frac{p}{p_{s}^{*}}}} .
$$


For the weak solution, we mean the one satisfying the given definition.

Definition 2.1 We say that $u \in W_{0}^{s, p}(\Omega)$ is a (weak) solution of problem (1.1) if

$$
\left(a+b[u]_{s, p}^{p}\right) L(u, v)=\lambda \int_{\Omega}|u|^{q-2} u v \ln |u|^{2} d x+\int_{\Omega}|u|^{p_{s}^{*}-2} u v d x,
$$

where

$$
L(u, v):=L(u, v) \iint_{\mathbb{R}^{2 N}} \frac{|u(x)-u(y)|^{p-2}(u(x)-u(y))}{|x-y|^{N+s p}}(v(x)-v(y)) d x d y
$$

for any $v \in W_{0}^{s, p}(\Omega)$.

The corresponding energy functional $I_{b}^{\lambda}: W_{0}^{s, p}(\Omega) \rightarrow \mathbb{R}$ to problem (1.1) is defined by

$$
I_{b}^{\lambda}(u)=\frac{a}{p}[u]_{s, p}^{p}+\frac{b}{2 p}[u]_{s, p}^{2 p}+\frac{2 \lambda}{q^{2}} \int_{\Omega}|u|^{q} d x-\frac{\lambda}{q} \int_{\Omega}|u|^{q} \ln |u|^{2} d x-\frac{1}{p_{s}^{*}} \int_{\Omega}|u|^{p_{s}^{*}} d x .
$$

It is easy to see that $I_{b}^{\lambda}$ belongs to $C^{1}\left(W_{0}^{s, p}(\Omega), \mathbb{R}\right)$ and the critical points of $I_{b}^{\lambda}$ are the solutions of (1.1). Furthermore, if we write

$$
u^{+}(x)=\max \{u(x), 0\} \text { and } u^{-}(x)=\min \{u(x), 0\}
$$

for $u \in W_{0}^{s, p}(\Omega)$, then every solution $u \in W_{0}^{s, p}(\Omega)$ of problem (1.1) with the property that $u^{ \pm} \neq 0$ is a sign-changing solution of problem (1.1).

It is noticed that if $u^{ \pm} \not \equiv 0$

$$
\begin{aligned}
& I_{b}^{\lambda}(u)=I_{b}^{\lambda}\left(u^{+}\right)+I_{b}^{\lambda}\left(u^{-}\right)+\frac{b}{p}\left\|u^{+}\right\|^{p}\left\|u^{-}\right\|^{p}, \\
& \left\langle\left(I_{b}^{\lambda}\right)^{\prime}(u), u^{+}\right\rangle=\left\langle\left(I_{b}^{\lambda}\right)^{\prime}\left(u^{+}\right), u^{+}\right\rangle+b\left\|u^{+}\right\|^{p}\left\|u^{-}\right\|^{p}, \\
& \left\langle\left(I_{b}^{\lambda}\right)^{\prime}(u), u^{-}\right\rangle=\left\langle\left(I_{b}^{\lambda}\right)^{\prime}\left(u^{-}\right), u^{-}\right\rangle+b\left\|u^{+}\right\|^{p}\left\|u^{-}\right\|^{p} .
\end{aligned}
$$

Our goal in this paper is then to seek the least energy sign-changing solutions of problem (1.1). As we know, there are some very interesting studies, which studied the existence and multiplicity of sign-changing solutions for the following problem:

$$
-\Delta u+V(x) u=f(x, u), \quad x \in \Omega,
$$

where $\Omega$ is an open subset of $\mathbb{R}^{N}$. However, these methods of seeking sign-changing solutions heavily rely on the following decompositions:

$$
\begin{aligned}
J(u) & =J\left(u^{+}\right)+J\left(u^{-}\right), \\
\left\langle J^{\prime}(u), u^{+}\right\rangle & =\left\langle J^{\prime}\left(u^{+}\right), u^{+}\right\rangle,\left\langle J^{\prime}(u), u^{-}\right\rangle=\left\langle J^{\prime}\left(u^{-}\right), u^{-}\right\rangle,
\end{aligned}
$$


where $J$ is the energy functional of (2.4) given by

$$
J(u)=\frac{1}{2} \int_{\Omega}\left(|\nabla u|^{2}+V(x) u^{2}\right) d x-\int_{\Omega} F(x, u) d x .
$$

However, if $b>0$, the energy functional $I_{b}^{\lambda}$ does not possess the same decompositions as (2.7) and (2.8). In fact, a straightforward computation yields that

$$
\begin{aligned}
& I_{b}^{\lambda}(u)>I_{b}^{\lambda}\left(u^{+}\right)+I_{b}^{\lambda}\left(u^{-}\right), \\
& \left\langle\left(I_{b}^{\lambda}\right)^{\prime}(u), u^{+}\right\rangle>\left\langle\left(I_{b}^{\lambda}\right)^{\prime}\left(u^{+}\right), u^{+}\right\rangle \text {and }\left\langle\left(I_{b}^{\lambda}\right)^{\prime}(u), u^{-}\right\rangle>\left\langle\left(I_{b}^{\lambda}\right)^{\prime}\left(u^{-}\right), u^{-}\right\rangle
\end{aligned}
$$

for $u^{ \pm} \neq 0$. Therefore, the method to obtain sign-changing solutions for the local problem (2.6) do not seem applicable to problem (1.1). In this paper, we follow the approach in [5] by defining the following constrained set

$$
\mathcal{M}_{b}^{\lambda}=\left\{u \in W_{0}^{s, p}(\Omega), u^{ \pm} \neq 0 \text { and }\left\langle\left(I_{b}^{\lambda}\right)^{\prime}(u), u^{+}\right\rangle=\left\langle\left(I_{b}^{\lambda}\right)^{\prime}(u), u^{-}\right\rangle=0\right\}
$$

and considering a minimization problem of $I_{b}^{\lambda}$ on $\mathcal{M}_{b}^{\lambda}$. Indeed, by using the parametric method and implicit theorem, Shuai [38] proved $\mathcal{M}_{b}^{\lambda} \neq \varnothing$ in the absence of the nonlocal term. However, the nonlocal term in problem (1.1), consisting of the biharmonic operator and the nonlocal term will cause some difficulties. Roughly speaking, compared to the general Kirchhoff type problem (1.2), decompositions (2.7) and (2.8) corresponding to $I_{b}^{\lambda}$ are much more complicated. This results in some technical difficulties during the proof of the nonempty of $\mathcal{M}_{b}^{\lambda}$. Moreover, we find that the parametric method and implicit theorem are not applicable for problem (1.1) due to the complexity of the nonlocal term there. Therefore, our proof takes a different route which is inspired by [1], namely, we make use of a modified Miranda's theorem (see [33]). We are also able to prove that the minimizer of the constrained problem is also a signchanging solution via the quantitative deformation lemma and degree theory. We can now present our first main result.

Theorem 2.1 There exists $\lambda^{*}>0$ such that for all $\lambda \geq \lambda^{*}$, problem (1.1) has a least energy sign-changing solution $u_{b} \in \mathcal{M}_{b}^{\lambda}$ with precisely two nodal domains such that $I_{b}^{\lambda}\left(u_{b}\right)=\inf _{u \in \mathcal{M}_{b}^{\lambda}} I_{b}^{\lambda}(u)$.

Another goal of this paper is to establish the so-called energy doubling property (cf. [45]), i.e., the energy of any sign-changing solution of problem (1.1) is strictly larger than twice the ground state energy. For the semilinear equation problem (2.6), the conclusion is trivial. Indeed, if we denote the Nehari manifold associated to problem (2.6) by

$$
\mathcal{N}=\left\{u \in W_{0}^{s, p}(\Omega) \backslash\{0\} \mid\left\langle J^{\prime}(u), u\right\rangle=0\right\}
$$

and define

$$
c=\inf _{u \in \mathcal{N}} J(u)
$$


then it is easy to verify that $u^{ \pm} \in \mathcal{N}$ for any sign-changing solution $u \in W_{0}^{s, p}(\Omega)$ to problem (2.6). We can deduce that

$$
J(w)=J\left(w^{+}\right)+J\left(w^{-}\right) \geq 2 c .
$$

We may point out that the minimizer of (2.10) is indeed a ground state solution of problem (2.6) and $c>0$ is the least energy of all weak solutions of problem (2.6). Therefore, by (2.11), it follows that the energy of any sign-changing solution of problem (2.6) is larger than twice the least energy. When $b>0$, a similar result was obtained by Shuai [38] in a bounded domain $\Omega$. We are also interested in that whether property (2.11) is still true for problem (1.1). To answer this question, we have the following result:

Theorem 2.2 There exists $\lambda^{* *}>0$ such that for all $\lambda \geq \lambda^{* *}$, the $c^{*}:=$ $\inf _{u \in \mathcal{N}_{b}^{\lambda}} I_{b}^{\lambda}(u)>0$ is achieved and $I_{b}^{\lambda}(u)>2 c^{*}$, where $\mathcal{N}_{b}^{\lambda}=\left\{u \in W_{0}^{s, p}(\Omega) \backslash\{0\} \mid\right.$ $\left.\left\langle\left(I_{b}^{\lambda}\right)^{\prime}(u), u\right\rangle=0\right\}$ and $u$ is the least energy sign-changing solution obtained in Theorem 2.1. In particular, $c^{*}>0$ is achieved either by a positive or a negative function.

It is obvious that the energy of the sign-changing solution $u_{b}$ obtained in Theorem 2.1 depends on $b$. In the following, we give a convergence property of $u_{b}$ as $b \rightarrow 0$, which reflects some relationship between $b>0$ and $b=0$ for problem (1.1).

Theorem 2.3 For any sequence $\left\{b_{n}\right\}$ with $b_{n} \rightarrow 0$ as $n \rightarrow \infty$, there exists a subsequence, still denoted by $\left\{b_{n}\right\}$, such that $\left\{u_{n}\right\}$ converges to $u_{0}$ strongly in $W_{0}^{s, p}(\Omega)$ as $n \rightarrow \infty$, where $u_{0}$ is a least energy sign-changing solution to the following problem

$$
\begin{cases}a(-\Delta)_{p}^{s} u=\lambda|u|^{q-2} u \ln |u|^{2}+|u|_{s}^{p_{s}^{*}-2} u & \text { in } \Omega, \\ u=0 & \text { in } \mathbb{R}^{N} \backslash \Omega .\end{cases}
$$

The plan of this paper is as follows: Sect. 2 covers the proof of the achievement of least energy for the constraint problem (1.1), Sect. 3 is devoted to the proofs of our main theorems.

Throughout this paper, we use standard notations. For simplicity, we use " $\rightarrow$ " and " $\rightarrow$ " to denote the strong and weak convergence in the related function space respectively. Various positive constants are denoted by $C$ and $C_{i}$. We use ":=" to denote definitions and $B_{r}(x):=\left\{y \in \mathbb{R}^{N}|| x-y \mid<r\right\}$. We denote a subsequence of a sequence $\left\{u_{n}\right\}_{n}$ as $\left\{u_{n}\right\}_{n}$ to simplify the notation unless specified.

\section{Some technical lemmas}

Now, fixed $u \in W_{0}^{s, p}(\Omega)$ with $u^{ \pm} \neq 0$, we define function $\psi_{u}:[0, \infty) \times[0, \infty) \rightarrow \mathbb{R}$ and mapping $T_{u}:[0, \infty) \times[0, \infty) \rightarrow \mathbb{R}^{2}$ by

$$
\psi_{u}(\alpha, \beta)=I_{b}^{\lambda}\left(\alpha u^{+}+\beta u^{-}\right)
$$


and

$$
T_{u}(\alpha, \beta)=\left(\left\langle\left(I_{b}^{\lambda}\right)^{\prime}\left(\alpha u^{+}+\beta u^{-}\right), \alpha u^{+}\right\rangle,\left\langle\left(I_{b}^{\lambda}\right)^{\prime}\left(\alpha u^{+}+\beta u^{-}\right), \beta u^{-}\right\rangle\right) .
$$

Lemma 3.1 For any $u \in W_{0}^{s, p}(\Omega)$ with $u^{ \pm} \neq 0$, then there is the unique maximum point pair $\left(\alpha_{u}, \beta_{u}\right)$ of the function $\psi_{u}$ such that $\alpha_{u} u^{+}+\beta_{u} u^{-} \in \mathcal{M}_{b}^{\lambda}$.

Proof Our proof will be divided into three steps.

Step 1 For any $u \in W_{0}^{s, p}(\Omega)$ with $u^{ \pm} \neq 0$, in the following, we will prove the existence of $\alpha_{u}$ and $\beta_{u}$.

From assumptions, we have that

$$
\lim _{t \rightarrow 0} \frac{|t|^{q-1} \ln |t|^{2}}{|t|^{p-1}}=0 \text { and } \lim _{t \rightarrow \infty} \frac{|t|^{q-1} \ln |t|^{2}}{|t|^{r-1}}=0
$$

for all $r \in\left(q, p_{s}^{*}\right)$. Then for any $\varepsilon>0$, there exists $C_{\varepsilon}>0$ such that

$$
|t|^{q-1} \ln |t|^{2} \leq \varepsilon|t|^{p-1}+C_{\varepsilon}|t|^{r-1} .
$$

Since $4 \leq 2 p<q<p_{s}^{*}$, it follows from (3.4) and the Sobolev embedding theorem that

$$
\begin{aligned}
\left\langle\left(I_{b}^{\lambda}\right)^{\prime}\left(\alpha u^{+}+\beta u^{-}\right), \alpha u^{+}\right\rangle= & a \alpha^{p}\left\|u^{+}\right\|^{p}+b \alpha^{2 p}\left\|u^{+}\right\|^{2 p}+b \alpha^{p} \beta^{p}\left\|u^{+}\right\|^{p}\left\|u^{-}\right\|^{p} \\
& -\lambda \int_{\Omega}\left|\alpha u^{+}\right|^{q} \ln \left|\alpha u^{+}\right|^{2} d x-\alpha^{p_{s}^{*}} \int_{\Omega}\left|u^{+}\right|^{p_{s}^{*}} d x \\
\geq & a \alpha^{p}\left\|u^{+}\right\|^{p}+b \alpha^{2 p}\left\|u^{+}\right\|^{2 p}+b \alpha^{p} \beta^{p}\left\|u^{+}\right\|^{p}\left\|u^{-}\right\|^{p} \\
& -\lambda \alpha^{p} \varepsilon \int_{\Omega}\left|u^{+}\right|^{p} d x-\lambda C_{\varepsilon} \alpha^{r} \int_{\Omega}\left|u^{+}\right|^{r} d x-\alpha^{p_{s}^{*}} \int_{\Omega}|u|^{p_{s}^{*}} d x \\
\geq & a \alpha^{p}\left\|u^{+}\right\|^{p}+b \alpha^{2 p}\left\|u^{+}\right\|^{2 p}-\lambda \alpha^{p} \varepsilon C_{1}\left\|u^{+}\right\|^{p} \\
& -\lambda C_{\varepsilon} \alpha^{r} C_{2}\left\|u^{+}\right\|^{r}-C_{3} \alpha^{p_{s}^{*}}\left\|u^{+}\right\|_{s}^{p_{s}^{*}} \\
= & \left(a-\lambda \varepsilon C_{1}\right) \alpha^{p}\left\|u^{+}\right\|^{p}+b \alpha^{2 p}\left\|u^{+}\right\|^{2 p}-\lambda C_{\varepsilon} \alpha^{r} C_{2}\left\|u^{+}\right\|^{r} \\
& -C_{3} \alpha^{p_{s}^{*}}\left\|u^{+}\right\|^{p_{s}^{*}} .
\end{aligned}
$$

Choose $\varepsilon>0$ such that $\left(a-\lambda \varepsilon C_{1}\right)>0$. Since $p_{s}^{*}, r>2 p$, we have that $\left\langle\left(I_{b}^{\lambda}\right)^{\prime}\left(\alpha u^{+}+\right.\right.$ $\left.\left.\beta u^{-}\right), \alpha u^{+}\right\rangle>0$ for $\alpha$ small enough and all $\beta \geq 0$.

Similarly, we obtain that $\left\langle\left(I_{b}^{\lambda}\right)^{\prime}\left(\alpha u^{+}+\beta u^{-}\right), \beta u^{-}\right\rangle>0$ for $\beta$ small enough and all $\alpha \geq 0$.

Therefore, there exists $\delta_{1}>0$ such that

$$
\left\langle\left(I_{b}^{\lambda}\right)^{\prime}\left(\delta_{1} u^{+}+\beta u^{-}\right), \delta_{1} u^{+}\right\rangle>0, \quad\left\langle\left(I_{b}^{\lambda}\right)^{\prime}\left(\alpha u^{+}+\delta_{1} u^{-}\right), \delta_{1} u^{-}\right\rangle>0
$$

for all $\alpha, \beta \geq 0$.

On the other hand, we can choose $\alpha=\delta_{2}^{*}>\delta_{1}$, if $\beta \in\left[\delta_{1}, \delta_{2}^{*}\right]$ and $\delta_{2}^{*}$ is large enough, it follows that 


$$
\begin{aligned}
\left\langle\left(I_{b}^{\lambda}\right)^{\prime}\left(\delta_{2}^{*} u^{+}+\beta u^{-}\right), \delta_{2}^{*} u^{+}\right\rangle \leq & a\left(\delta_{2}^{*}\right)^{p}\left\|u^{+}\right\|^{p}+b\left(\delta_{2}^{*}\right)^{2 p}\left\|u^{+}\right\|^{2 p}+b\left(\delta_{2}^{*}\right)^{2 p}\left\|u^{+}\right\|^{p}\left\|u^{-}\right\|^{p} \\
& -\left(\delta_{2}^{*}\right)^{p_{s}^{*}} \int_{\Omega}\left|u^{+}\right|^{p_{s}^{*}} d x \leq 0 .
\end{aligned}
$$

Similarly, we have that

$$
\begin{aligned}
\left\langle\left(I_{b}^{\lambda}\right)^{\prime}\left(\alpha u^{+}+\delta_{2}^{*} u^{-}\right), \delta_{2}^{*} u^{-}\right\rangle \leq & \left(\delta_{2}^{*}\right)^{p}\left\|u^{-}\right\|^{p}+b\left(\delta_{2}^{*}\right)^{2 p}\left\|u^{+}\right\|^{2 p}+b\left(\delta_{2}^{*}\right)^{2 p}\left\|u^{+}\right\|^{p}\left\|u^{-}\right\|^{p} \\
& -\left(\delta_{2}^{*}\right)^{p_{s}^{*}} \int_{\Omega}\left|u^{-}\right|^{p_{s}^{*}} d x \leq 0 .
\end{aligned}
$$

Let $\delta_{2}>\delta_{2}^{*}$ be large enough, we obtain that

$$
\left\langle\left(I_{b}^{\lambda}\right)^{\prime}\left(\delta_{2}^{*} u^{+}+\beta u^{-}\right), \delta_{2}^{*} u^{+}\right\rangle<0 \text { and }\left\langle\left(I_{b}^{\lambda}\right)^{\prime}\left(\alpha u^{+}+\delta_{2}^{*} u^{-}\right), \delta_{2}^{*} u^{-}\right\rangle<0
$$

for all $\alpha, \beta \in\left[\delta_{1}, \delta_{2}\right]$.

Combining (3.5) and (3.6) with Miranda's theorem [33], there exists $\left(\alpha_{u}, \beta_{u}\right) \in$ $(0,+\infty) \times(0,+\infty)$ such that $T_{u}(\alpha, \beta)=(0,0)$, i.e., $\alpha u^{+}+\beta u^{-} \in \mathcal{M}_{b}^{\lambda}$.

Step 2 In this step, we prove the uniqueness of the pair $\left(\alpha_{u}, \beta_{u}\right)$.

- Case $u \in \mathcal{M}_{b}^{\lambda}$.

If $u \in \mathcal{M}_{b}^{\lambda}$, we have that

$$
\left\|u^{+}\right\|^{p}+b\left\|u^{+}\right\|^{2 p}+b\left\|u^{+}\right\|^{p}\left\|u^{-}\right\|^{p}=\lambda \int_{\Omega}\left|u^{+}\right|^{q} \ln \left|u^{+}\right|^{2} d x+\int_{\Omega}\left|u^{+}\right|^{p_{s}^{*}} d x
$$

and

$$
\left\|u^{-}\right\|^{2}+b\left\|u^{-}\right\|^{2 p}+b\left\|u^{+}\right\|^{p}\left\|u^{-}\right\|^{p}=\lambda \int_{\Omega}\left|u^{-}\right|^{q} \ln \left|u^{-}\right|^{2} d x+\int_{\Omega}\left|u^{-}\right|^{p_{s}^{*}} d x .
$$

We show that $\left(\alpha_{u}, \beta_{u}\right)=(1,1)$ is the unique pair of numbers such that $\alpha_{u} u^{+}+\beta_{u} u^{-} \in$ $\mathcal{M}_{b}^{\lambda}$.

Let $\left(\alpha_{0}, \beta_{0}\right)$ be a pair of numbers such that $\alpha_{0} u^{+}+\beta_{0} u^{-} \in \mathcal{M}_{b}^{\lambda}$ with $0<\alpha_{0} \leq \beta_{0}$. Hence, one has that

$$
\begin{aligned}
\alpha_{0}^{p}\left\|u^{+}\right\|^{p}+b \alpha_{0}^{2 p}\left\|u^{+}\right\|^{2 p}+b \alpha_{0}^{p} \beta_{0}^{p}\left\|u^{+}\right\|^{p}\left\|u^{-}\right\|^{p} & =\lambda \int_{\Omega}\left|\alpha_{0} u^{+}\right|^{q} \ln \left|\alpha_{0} u^{+}\right|^{2} d x \\
& +\alpha_{0}^{p_{s}^{*}} \int_{\Omega}\left|u^{+}\right|^{p_{s}^{*}} d x
\end{aligned}
$$


and

$$
\begin{aligned}
\beta_{0}^{p}\left\|u^{-}\right\|^{p}+b \beta_{0}^{2 p}\left\|u^{-}\right\|^{2 p}+b \alpha_{0}^{p} \beta_{0}^{p}\left\|u^{+}\right\|^{p}\left\|u^{-}\right\|^{p} & =\lambda \int_{\Omega}\left|\beta_{0} u^{-}\right|^{q} \ln \left|\beta_{0} u^{-}\right|^{2} d x \\
& +\beta_{0}^{p_{s}^{*}} \int_{\Omega}\left|u^{-}\right|^{p_{s}^{*}} d x
\end{aligned}
$$

According to $0<\alpha_{0} \leq \beta_{0}$ and (3.10), we have that

$$
\frac{\left\|u^{-}\right\|^{p}}{\beta_{0}^{p}}+b\left\|u^{-}\right\|^{2 p}+b\left\|u^{+}\right\|^{p}\left\|u^{-}\right\|^{p} \geq \lambda \int_{\Omega} \frac{\left|\beta_{0} u^{-}\right|^{q} \ln \left|\beta_{0} u^{-}\right|^{2}}{\beta_{0}^{2 p}} d x+\beta_{0}^{p_{s}^{*}-2 p} \int_{\Omega}\left|u^{-}\right|^{p_{s}^{*}} d x
$$

If $\beta_{0}>1$, by (3.8) and (3.11), one has that

$$
\begin{aligned}
& 0>\left(\frac{1}{\beta_{0}^{p}}-1\right)\left\|u^{-}\right\|^{p} \geq \lambda \int_{\Omega}\left[\frac{\left|\beta_{0} u^{-}\right|^{q} \ln \left|\beta_{0} u^{-}\right|^{2}}{\beta_{0}^{2 p}}-\left|u^{-}\right|^{q} \ln \left|u^{-}\right|^{2}\right] d x \\
& +\left(\beta_{0}^{p_{s}^{*}-2 p}-1\right) \int_{\Omega}\left|u^{-}\right|^{p_{s}^{*}} d x \\
& \geq\left(\beta_{0}^{p_{s}^{*}-2 p}-1\right) \int_{\Omega}\left|u^{-}\right|^{p_{s}^{*}} d x>0 .
\end{aligned}
$$

This is a contradiction. Therefore, we conclude that $0<\alpha_{0} \leq \beta_{0} \leq 1$.

Similarly, by (3.9) and $0<\alpha_{0} \leq \beta_{0}$, we have that

$$
\begin{aligned}
\left(\frac{1}{\alpha_{0}^{p}}-1\right)\left\|u^{+}\right\|^{p} \leq & \lambda \int_{\Omega}\left[\frac{\left|\alpha_{0} u^{+}\right|^{q} \ln \left|\alpha_{0} u^{+}\right|^{2}}{\alpha_{0}^{2 p}}-\left|u^{+}\right|^{q} \ln \left|u^{+}\right|^{2}\right] d x \\
& +\left(\beta_{0}^{p_{s}^{*}-2 p}-1\right) \int_{\Omega}\left|u^{+}\right|^{p_{s}^{*}} d x .
\end{aligned}
$$

This fact implies that $\alpha_{0} \geq 1$. Consequently, $\alpha_{0}=\beta_{0}=1$.

- Case $u \notin \mathcal{M}_{b}^{\lambda}$.

Suppose that there exist $\left(\alpha_{1}, \beta_{1}\right),\left(\alpha_{2}, \beta_{2}\right)$ such that

$$
\omega_{1}=\alpha_{1} u^{+}+\beta_{1} u^{-} \in \mathcal{M}_{b}^{\lambda} \text { and } \omega_{2}=\alpha_{2} u^{+}+\beta_{2} u^{-} \in \mathcal{M}_{b}^{\lambda}
$$

Hence

$$
\omega_{2}=\left(\frac{\alpha_{2}}{\alpha_{1}}\right) \alpha_{1} u^{+}+\left(\frac{\beta_{2}}{\beta_{1}}\right) \beta_{1} u^{-}=\left(\frac{\alpha_{2}}{\alpha_{1}}\right) \omega_{1}^{+}+\left(\frac{\beta_{2}}{\beta_{1}}\right) \omega_{1}^{-} \in \mathcal{M}_{b}^{\lambda} .
$$

By $\omega_{1} \in \mathcal{M}_{b}^{\lambda}$, one has that

$$
\frac{\alpha_{2}}{\alpha_{1}}=\frac{\beta_{2}}{\beta_{1}}=1
$$


Hence, $\alpha_{1}=\alpha_{2}, \beta_{1}=\beta_{2}$.

Step 3 In this step, we will prove that $\left(\alpha_{u}, \beta_{u}\right)$ is the unique maximum point of $\psi_{u}$ on $[0, \infty) \times[0, \infty)$.

First, it is easy to see that

$$
2 \tau^{q}-q \tau^{q} \ln |\tau|^{2} \leq 2 \text { for all } \tau \in(0, \infty)
$$

Let $\Omega^{+}=\{x \in \Omega: u(x)>0\}$ and $\Omega^{-}=\{x \in \Omega: u(x)<0\}, u \in W_{0}^{s, p}(\Omega)$ with $u^{ \pm} \neq 0$, we have that

$$
\begin{aligned}
\int_{\Omega}\left|\alpha u^{+}+\beta u^{-}\right|^{q} \ln \left|\alpha u^{+}+\beta u^{-}\right|^{2} d x= & \int_{\Omega^{+}}\left|\alpha u^{+}+\beta u^{-}\right|^{q} \ln \left|\alpha u^{+}+\beta u^{-}\right|^{2} d x \\
& +\int_{\Omega^{-}}\left|\alpha u^{+}+\beta u^{-}\right|^{q} \ln \left|\alpha u^{+}+\beta u^{-}\right|^{2} d x \\
= & \int_{\Omega^{+}}\left|\alpha u^{+}\right|^{q} \ln \left|\alpha u^{+}\right|^{2} d x+\int_{\Omega^{-}}\left|\beta u^{-}\right|^{q} \ln \left|\beta u^{-}\right|^{2} d x \\
& =\int_{\Omega}\left[\left|\alpha u^{+}\right|^{q} \ln \left|\alpha u^{+}\right|^{2}+\left|\beta u^{-}\right|^{q} \ln \left|\beta u^{-}\right|^{2}\right] d x
\end{aligned}
$$

Combining (3.12) and (3.13), we have that

$$
\begin{aligned}
\psi_{u}(\alpha, \beta)= & I_{b}^{\lambda}\left(\alpha u^{+}+\beta u^{-}\right) \\
= & \frac{1}{p}\left\|\alpha u^{+}+\beta u^{-}\right\|^{p}+\frac{b}{2 p}\left\|\alpha u^{+}+\beta u^{-}\right\|^{2 p}+\frac{2 \lambda}{q^{2}} \int_{\Omega}\left|\alpha u^{+}+\beta u^{-}\right|^{q} d x \\
& -\frac{\lambda}{q} \int_{\Omega}\left|\alpha u^{+}+\beta u^{-}\right|^{q} \ln \left|\alpha u^{+}+\beta u^{-}\right|^{2} d x-\frac{1}{p_{s}^{*}} \int_{\Omega}\left|\alpha u^{+}+\beta u^{-}\right|^{p_{s}^{*}} d x \\
= & \frac{\alpha^{p}}{p}\left\|u^{+}\right\|^{p}+\frac{\beta^{p}}{p}\left\|u^{-}\right\|^{p}+\frac{b \alpha^{2 p}}{2 p}\left\|u^{+}\right\|^{2 p}+\frac{b \beta^{2 p}}{2 p}\left\|u^{-}\right\|^{2 p}+\frac{b \alpha^{p} \beta^{p}}{p}\left\|u^{+}\right\|^{p}\left\|u^{-}\right\|^{p} \\
& +\frac{\lambda}{q^{2}} \int_{\Omega}\left(2\left|\alpha u^{+}\right|^{q}-q\left|\alpha u^{+}\right|^{q} \ln \left|\alpha u^{+}\right|^{2}\right) d x \\
& +\frac{\lambda}{q^{2}} \int_{\Omega}\left(2\left|\beta u^{-}\right|^{q}-q\left|\beta u^{-}\right|^{q} \ln \left|\beta u^{-}\right|^{2}\right) d x \\
& -\frac{\alpha^{p_{s}^{*}}}{p_{s}^{*}} \int_{\Omega}\left|u^{+}\right|^{p_{s}^{*}} d x-\frac{\beta^{p_{s}^{*}}}{p_{s}^{*}} \int_{\Omega}\left|u^{-}\right|^{p_{s}^{*}} d x \\
\leq & \frac{\alpha^{p}}{p}\left\|u^{+}\right\|^{p}+\frac{\beta^{p}}{p}\left\|u^{-}\right\|^{p}+\frac{b \alpha^{2 p}}{2 p}\left\|u^{+}\right\|^{2 p}+\frac{b \beta^{2 p}}{2 p}\left\|u^{-}\right\|^{2 p}+\frac{b \alpha^{p} \beta^{p}}{p}\left\|u^{+}\right\|^{p}\left\|u^{-}\right\|^{p} \\
& +\frac{4}{q^{2}} \lambda|\Omega|-\frac{\alpha^{p_{s}^{*}}}{p_{s}^{*}} \int_{\Omega}\left|u^{+}\right|^{p_{s}^{*}} d x-\frac{\beta^{p_{s}^{*}}}{p_{s}^{*}} \int_{\Omega}\left|u^{-}\right|^{p_{s}^{*}} d x
\end{aligned}
$$

which implies that $\lim _{|(\alpha, \beta)| \rightarrow \infty} \psi_{u}(\alpha, \beta)=-\infty$ since $p_{s}^{*}>2 p$. Hence, $\left(\alpha_{u}, \beta_{u}\right)$ is the unique critical point of $\psi_{u}$ in $[0, \infty) \times[0, \infty)$. So it is sufficient to check that a maximum point cannot be achieved on the boundary of $[0, \infty) \times[0, \infty)$. By contradiction, we suppose that $\left(0, \beta_{0}\right)$ is a maximum point of $\psi_{u}$ with $\beta_{0} \geq 0$. Then, we have that 


$$
\begin{aligned}
\psi_{u}\left(\alpha, \beta_{0}\right)= & \frac{\alpha^{p}}{p}\left\|u^{+}\right\|^{p}+\frac{b \alpha^{2 p}}{2 p}\left\|u^{+}\right\|^{2 p}+\frac{2 \alpha^{q} \lambda}{q^{2}} \int_{\Omega}\left|u^{+}\right|^{q} d x-\frac{\alpha^{q} \lambda}{q} \int_{\Omega}\left|u^{+}\right|^{q} \ln \left|\alpha u^{+}\right|^{2} d x \\
- & \frac{\alpha^{p_{s}^{*}}}{p_{s}^{*}} \int_{\Omega}\left|u^{+}\right|^{p_{s}^{*}} d x+\frac{\beta_{0}^{p}}{p}\left\|u^{-}\right\|^{p}+\frac{b \beta_{0}^{2 p}}{2 p}\left\|u^{-}\right\|^{2 p}+\frac{2 \beta^{q} \lambda}{q^{2}} \int_{\Omega}\left|u^{-}\right|^{q} d x \\
& -\frac{\beta^{q} \lambda}{q} \int_{\Omega}\left|u^{-}\right|^{q} \ln \left|\beta u^{-}\right|^{2} d x-\frac{\beta^{p_{s}^{*}}}{p_{s}^{*}} \int_{\Omega}\left|u^{-}\right|^{p_{s}^{*}} d x \\
& +\frac{b \alpha^{p} \beta_{0}^{p}}{p}\left\|u^{+}\right\|^{p}\left\|u^{-}\right\|^{p} .
\end{aligned}
$$

Therefore, it is obvious that

$$
\begin{aligned}
\left(\psi_{u}\right)_{\alpha}^{\prime}\left(\alpha, \beta_{0}\right)= & \alpha^{p-1}\left\|u^{+}\right\|^{p}+b \alpha^{2 p-1}\left\|u^{+}\right\|^{2 p}+b \alpha^{p-1} \beta_{0}^{p}\left\|u^{+}\right\|^{p}\left\|u^{-}\right\|^{p} \\
& +\frac{2 \alpha^{q-1} \lambda}{q} \int_{\Omega}\left|u^{+}\right|^{q} d x-\alpha^{q-1} \int_{\Omega}\left|u^{+}\right|^{q} \ln \left|\alpha u^{+}\right|^{2} d x-\alpha^{p_{s}^{*}-1} \int_{\Omega}\left|u^{+}\right|^{p_{s}^{*}} d x \\
& >0
\end{aligned}
$$

if $\alpha$ is small enough. That is, $\psi_{u}$ is an increasing function with respect to $\alpha$ if $\alpha$ is small enough. This yields the contradiction. Similarly, $\psi_{u}$ can not achieve its global maximum on $(\alpha, 0)$ with $\alpha \geq 0$.

Lemma 3.2 For any $u \in W_{0}^{s, p}(\Omega)$ with $u^{ \pm} \neq 0$ such that $\left\langle\left(I_{b}^{\lambda}\right)^{\prime}(u), u^{ \pm}\right\rangle \leq 0$. Then, the unique maximum point of $\psi_{u}$ on $[0, \infty) \times[0, \infty)$ satisfies $0<\alpha_{u}, \beta_{u} \leq 1$.

Proof Without loss of generality, let $\alpha_{u} \geq \beta_{u}>0$.

On the one hand, by $\alpha_{u} u^{+}+\beta_{u} u^{-} \in \mathcal{M}_{b}^{\lambda}$, we have

$$
\begin{aligned}
a \alpha_{u}^{p}\left\|u^{+}\right\|^{p}+b \alpha_{u}^{2 p}\left\|u^{+}\right\|^{2 p}+b \alpha_{u}^{2 p}\left\|u^{+}\right\|^{p}\left\|u^{-}\right\|^{p} & =\lambda \alpha_{u}^{q} \int_{\Omega}\left|u^{+}\right|^{q} \ln \left|\alpha_{u} u^{+}\right|^{2} d x \\
& -\alpha_{u}^{p_{s}^{*}} \int_{\Omega}\left|u^{+}\right|^{p_{s}^{*}} d x
\end{aligned}
$$

On the other hand, by $\left\langle\left(I_{b}^{\lambda}\right)^{\prime}(u), u^{+}\right\rangle \leq 0$, we have

$$
a\left\|u^{+}\right\|^{p}+b\left\|u^{+}\right\|^{2 p}+b\left\|u^{+}\right\|^{p}\left\|u^{-}\right\|^{p} \leq \lambda \int_{\Omega}\left|u^{+}\right|^{q} \ln \left|u^{+}\right|^{2} d x+\int_{\Omega}\left|u^{+}\right|^{p_{s}^{*}} d x .
$$

So, according to (3.14) and (3.15), we have that

$$
\begin{aligned}
\left(\frac{1}{\alpha_{u}^{p}}-1\right) a\left\|u^{+}\right\|^{p} \geq & \lambda \int_{\Omega}\left[\alpha_{u}^{q-2 p}\left|u^{+}\right|^{q} \ln \left|\alpha_{u} u^{+}\right|^{2}-\left|u^{+}\right|^{q} \ln \left|u^{+}\right|^{2}\right] d x \\
& +\left(\alpha_{u}^{p_{s}^{*}-2 p}-1\right) \int_{\Omega}\left|u^{+}\right|^{p_{s}^{*}} d x
\end{aligned}
$$


If $\alpha_{u} \geq 1$, one has

$$
\int_{\Omega}\left[\alpha_{u}^{q-2 p}\left|u^{+}\right|^{q} \ln \left|\alpha_{u} u^{+}\right|^{2}-\left|u^{+}\right|^{q} \ln \left|u^{+}\right|^{2}\right] d x \geq 0 .
$$

This fact together with (3.16), we have

$$
0>\left(\frac{1}{\alpha_{u}^{p}}-1\right) a\left\|u^{+}\right\|^{p} \geq\left(\alpha_{u}^{p_{s}^{*}-2 p}-1\right) \int_{\Omega}\left|u^{+}\right|^{p_{s}^{*}} d x>0 .
$$

This is a contradiction. Thus, we conclude that $\alpha_{u} \leq 1$. Thus, we have that $0<$ $\alpha_{u}, \beta_{u} \leq 1$

Lemma 3.3 Let $c_{b}^{\lambda}=\inf _{u \in \mathcal{M}_{b}^{\lambda}} I_{b}^{\lambda}(u)$, then we have that $\lim _{\lambda \rightarrow \infty} c_{b}^{\lambda}=0$.

Proof For any $u \in \mathcal{M}_{b}^{\lambda}$, we have

$$
a\left\|u^{ \pm}\right\|^{p}+b\left\|u^{ \pm}\right\|^{2 p}+b\left\|u^{+}\right\|^{p}\left\|u^{-}\right\|^{p}=\lambda \int_{\Omega}\left|u^{ \pm}\right|^{q} \ln \left|u^{ \pm}\right|^{2} d x+\int_{\Omega}\left|u^{ \pm}\right|^{p_{s}^{*}} d x .
$$

Then, by (3.4) and the Sobolev theorem, we have that

$$
\begin{aligned}
a\left\|u^{ \pm}\right\|^{p} & \leq \lambda \int_{\Omega}\left|u^{ \pm}\right|^{q} \ln \left|u^{ \pm}\right|^{2} d x+\int_{\Omega}\left|u^{ \pm}\right|^{p_{s}^{*}} d x \\
& \leq \lambda \varepsilon C_{1}\left\|u^{ \pm}\right\|^{p}+\lambda C_{\varepsilon} C_{2}\left\|u^{ \pm}\right\|^{r}+C_{3}\left\|u^{ \pm}\right\|^{p_{s}^{*}} .
\end{aligned}
$$

Thus, we get

$$
\left(1-\lambda \varepsilon C_{1}\right) a\left\|u^{ \pm}\right\|^{p} \leq \lambda C_{\varepsilon} C_{2}\left\|u^{ \pm}\right\|^{r}+C_{3}\left\|u^{ \pm}\right\|^{p_{s}^{*}}
$$

Choosing $\varepsilon$ small enough such that $1-\lambda \varepsilon C_{1}>0$, since $r, p_{s}^{*}>p$, there exists $\rho>0$ such that

$$
\left\|u^{ \pm}\right\| \geq \rho \text { for all } u \in \mathcal{M}_{b}^{\lambda}
$$

On the other hand, for any $u \in \mathcal{M}_{b}^{\lambda}$, it is obvious that $\left\langle\left(I_{b}^{\lambda}\right)^{\prime}(u), u\right\rangle=0$. Then, we have

$$
\begin{aligned}
I_{b}^{\lambda}(u)= & I_{b}^{\lambda}(u)-\frac{1}{q}\left\langle\left(I_{b}^{\lambda}\right)^{\prime}(u), u\right\rangle \\
= & \left(\frac{1}{p}-\frac{1}{q}\right) a\|u\|^{p}+\left(\frac{1}{2 p}-\frac{1}{q}\right) b\|u\|^{2 p}+\left(\frac{1}{q}-\frac{1}{p_{s}^{*}}\right) \int_{\Omega}|u|^{p_{s}^{*}} d x \\
& +\frac{2 \lambda}{q^{2}} \int_{\Omega}|u|^{q} d x \geq\left(\frac{1}{p}-\frac{1}{q}\right) a\|u\|^{p} .
\end{aligned}
$$


From above discussions, we have that $I_{b}^{\lambda}(u)>0$ for all $u \in \mathcal{M}_{b}^{\lambda}$. Therefore, $I_{b}^{\lambda}$ is bounded below on $\mathcal{M}_{b}^{\lambda}$, that is $c_{b}^{\lambda}=\inf _{u \in \mathcal{M}_{b}^{\lambda}} I_{b}^{\lambda}(u)$ is well defined.

Let $u \in W_{0}^{s, p}(\Omega)$ with $u^{ \pm} \neq 0$ be fixed. By Lemma 3.1, for each $\lambda>0$, there exist $\alpha_{\lambda}, \beta_{\lambda}>0$ such that $\alpha_{\lambda} u^{+}+\beta_{\lambda} u^{-} \in \mathcal{M}_{b}^{\lambda}$. By using Lemma 3.1 again, we have that

$$
\begin{aligned}
0 \leq c_{b}^{\lambda} & =\inf _{u \in \mathcal{M}_{b}^{\lambda}} I_{b}^{\lambda}(u) \leq I_{b}^{\lambda}\left(\alpha_{\lambda} u^{+}+\beta_{\lambda} u^{-}\right) \\
& \leq \frac{1}{p} a\left\|\alpha_{\lambda} u^{+}+\beta_{\lambda} u^{-}\right\|^{p}+\frac{b}{2 p}\left\|\alpha_{\lambda} u^{+}+\beta_{\lambda} u^{-}\right\|^{2 p} \\
& \leq a \alpha_{\lambda}^{p}\left\|u^{+}\right\|^{p}+a \beta_{\lambda}^{p}\left\|u^{-}\right\|^{p}+p b \alpha_{\lambda}^{2 p}\left\|u^{+}\right\|^{2 p}+p b \beta_{\lambda}^{2 p}\left\|u^{-}\right\|^{2 p}
\end{aligned}
$$

To our end, we just prove that $\alpha_{\lambda} \rightarrow 0$ and $\beta_{\lambda} \rightarrow 0$ as $\lambda \rightarrow \infty$.

Let

$$
\mathcal{T}_{u}=\left\{\left(\alpha_{\lambda}, \beta_{\lambda}\right) \in[0, \infty) \times[0, \infty): T_{u}\left(\alpha_{\lambda}, \beta_{\lambda}\right)=(0,0), \lambda>0\right\},
$$

where $T_{u}$ is defined as (3.2). By (3.3), we have that

$$
\begin{aligned}
\alpha_{\lambda}^{p_{s}^{*}} & \int_{\Omega}\left|u^{+}\right|^{p_{s}^{*}} d x+\beta_{\lambda}^{p_{s}^{*}} \int_{\Omega}\left|u^{-}\right| p^{*} d x \\
\leq & \alpha_{\lambda}^{p_{s}^{*}} \int_{\Omega}\left|u^{+}\right|^{p_{s}^{*}} d x+\beta_{\lambda}^{p_{s}^{*}} \int_{\Omega}\left|u^{-}\right|^{p_{s}^{*}} d x+\lambda \alpha_{\lambda}^{q} \int_{\Omega}\left|u^{+}\right|^{q} \ln \left|u^{+}\right|^{2} d x \\
& +\lambda \beta_{\lambda}^{q} \int_{\Omega}\left|u^{+}\right|^{q} \ln \left|u^{+}\right|^{2} d x \\
= & \left\|\alpha_{\lambda} u^{+}+\beta_{\lambda} u^{-}\right\|^{p}+b\left\|\alpha_{\lambda} u^{+}+\beta_{\lambda} u^{-}\right\|^{2 p} \\
\leq & p \alpha_{\lambda}^{p}\left\|u^{+}\right\|^{p}+p \beta_{\lambda}^{p}\left\|u^{-}\right\|^{p}+2 p b \alpha_{\lambda}^{2 p}\left\|u^{+}\right\|^{2 p}+2 p b \beta_{\lambda}^{2 p}\left\|u^{-}\right\|^{2 p} .
\end{aligned}
$$

Hence, $\mathcal{T}_{u}$ is bounded since $2 p<p_{s}^{*}$. Let $\left\{\lambda_{n}\right\} \subset(0, \infty)$ be such that $\lambda_{n} \rightarrow \infty$ as $n \rightarrow \infty$. Then, there exist $\alpha_{0}$ and $\beta_{0}$ such that $\left(\alpha_{\lambda_{n}}, \beta_{\lambda_{n}}\right) \rightarrow\left(\alpha_{0}, \beta_{0}\right)$ as $n \rightarrow \infty$.

Now, we claim $\alpha_{0}=\beta_{0}=0$. Suppose, by contradiction, that $\alpha_{0}>0$ or $\beta_{0}>0$. By $\alpha_{\lambda_{n}} u^{+}+\beta_{\lambda_{n}} u^{-} \in \mathcal{M}_{b}^{\lambda_{n}}$, for any $n \in \mathbb{N}$, we have

$$
\begin{aligned}
& \left\|\alpha_{\lambda_{n}} u^{+}+\beta_{\lambda_{n}} u^{-}\right\|^{p}+b\left\|\alpha_{\lambda_{n}} u^{+}+\beta_{\lambda_{n}} u^{-}\right\|^{2 p} \\
& \quad=\lambda_{n} \int_{\Omega}\left|\alpha_{\lambda_{n}} u^{+}+\beta_{\lambda_{n}} u^{-}\right|^{q} \ln \left|\alpha_{\lambda_{n}} u^{+}+\beta_{\lambda_{n}} u^{-}\right|^{2} d x+\int_{\Omega}\left|\alpha_{\lambda_{n}} u^{+}+\beta_{\lambda_{n}} u^{-}\right|^{p_{s}^{*}} d x
\end{aligned}
$$

Thanks to $\alpha_{\lambda_{n}} u^{+} \rightarrow \alpha_{0} u^{+}$and $\beta_{\lambda_{n}} u^{-} \rightarrow \beta_{0} u^{+}$in $W_{0}^{s, p}(\Omega),(3.4)$ and the Lebesgue dominated convergence theorem, we have that

$$
\begin{aligned}
& \int_{\Omega}\left|\alpha_{\lambda_{n}} u^{+}+\beta_{\lambda_{n}} u^{-}\right|^{q} \ln \left|\alpha_{\lambda_{n}} u^{+}+\beta_{\lambda_{n}} u^{-}\right|^{2} d x \\
& \quad \rightarrow \int_{\Omega}\left|\alpha_{0} u^{+}+\beta_{0} u^{-}\right|^{q} \ln \left|\alpha_{0} u^{+}+\beta_{0} u^{-}\right|^{2} d x>0
\end{aligned}
$$


as $n \rightarrow \infty$. It follows from $\lambda_{n} \rightarrow \infty$ as $n \rightarrow \infty$ and $\left\{\alpha_{\lambda_{n}} u^{+}+\beta_{\lambda_{n}} u^{-}\right\}$is bounded in $W_{0}^{s, p}(\Omega)$ that we have a contradiction with equality (3.18). Hence, $\alpha_{0}=\beta_{0}=0$.

Hence, we conclude that $\lim _{\lambda \rightarrow \infty} c_{b}^{\lambda}=0$.

Lemma 3.4 There exists $\lambda^{*}>0$ such that for all $\lambda \geq \lambda^{*}$, the infimum $c_{b}^{\lambda}$ is achieved.

Proof By the definition of $c_{b}^{\lambda}$, there exists a sequence $\left\{u_{n}\right\} \subset \mathcal{M}_{b}^{\lambda}$ such that

$$
\lim _{n \rightarrow \infty} I_{b}^{\lambda}\left(u_{n}\right)=c_{b}^{\lambda}
$$

Obviously, $\left\{u_{n}\right\}$ is bounded in $W_{0}^{s, p}(\Omega)$. Then, up to a subsequence, still denoted by $\left\{u_{n}\right\}$, there exists $u \in W_{0}^{s, p}(\Omega)$ such that $u_{n} \rightarrow u$. Since the embedding $W_{0}^{s, p}(\Omega) \hookrightarrow$ $L^{t}(\Omega)$ is compact, for all $t \in\left(p, p_{s}^{*}\right)$, we have

$$
u_{n} \rightarrow u \text { in } L^{t}(\Omega), \quad u_{n} \rightarrow u \text { a.e. } x \in \Omega
$$

Hence

$$
\begin{aligned}
& u_{n}^{ \pm} \rightarrow u^{ \pm} \text {in } W_{0}^{s, p}(\Omega), \\
& u_{n}^{ \pm} \rightarrow u^{ \pm} \text {in } L^{t}(\Omega), \\
& u_{n}^{ \pm} \rightarrow u^{ \pm} \text {a.e. } x \in \Omega .
\end{aligned}
$$

By Lemma 3.1, we have that

$$
I_{b}^{\lambda}\left(\alpha u_{n}^{+}+\beta u_{n}^{-}\right) \leq I_{b}^{\lambda}\left(u_{n}\right)
$$

for all $\alpha, \beta \geq 0$.

On the one hand, the Vitali convergence theorem yields that

$$
\lim _{n \rightarrow \infty} \int_{\Omega}\left|u_{n}\right|^{q} \ln \left|u_{n}\right|^{2} d x \rightarrow \int_{\Omega}|u|^{q} \ln |u|^{2} d x .
$$

On the other hand, since $u_{n} \rightarrow u$ in $L^{q}(\Omega)$, we have

$$
\lim _{n \rightarrow \infty} \int_{\Omega}\left|u_{n}\right|^{q} d x \rightarrow \int_{\Omega}|u|^{q} d x .
$$

Then, by (3.19), (3.20), Brézis-Lieb lemma and the weak semicontinuity of norm, we have

$$
\begin{aligned}
& \liminf _{n \rightarrow \infty} I_{b}^{\lambda}\left(\alpha u_{n}^{+}+\beta u_{n}^{-}\right) \\
& \geq \frac{a \alpha^{p}}{p} \lim _{n \rightarrow \infty}\left(\left\|u_{n}^{+}-u^{+}\right\|^{p}+\left\|u^{+}\right\|^{p}\right)+\frac{a \beta^{p}}{p} \lim _{n \rightarrow \infty}\left(\left\|u_{n}^{-}-u^{-}\right\|^{p}+\left\|u^{-}\right\|^{p}\right) \\
& \quad+\frac{b \alpha^{2 p}}{2 p}\left[\lim _{n \rightarrow \infty}\left(\left\|u_{n}^{+}-u^{+}\right\|^{p}+\left\|u^{+}\right\|^{p}\right)\right]^{2}+\frac{b \beta^{2 p}}{2 p}\left[\lim _{n \rightarrow \infty}\left(\left\|u_{n}^{-}-u^{-}\right\|^{p}+\left\|u^{-}\right\|^{p}\right)\right]^{2}
\end{aligned}
$$




$$
\begin{aligned}
& -\frac{\alpha^{p_{s}^{*}}}{p_{s}^{*}} \lim _{n \rightarrow \infty}\left[\int_{\Omega}\left|u_{n}^{+}-u^{+}\right|^{p_{s}^{*}} d x+\int_{\Omega}\left|u^{+}\right|^{p_{s}^{*}} d x\right] \\
& -\frac{\beta^{p_{s}^{*}}}{p_{s}^{*}} \lim _{n \rightarrow \infty}\left[\int_{\Omega}\left|u_{n}^{-}-u^{-}\right|^{p_{s}^{*}} d x+\int_{\Omega}\left|u^{-}\right|^{p_{s}^{*}} d x\right] \\
& +\frac{2 \lambda}{q^{2}} \int_{\Omega}|u|^{q} d x-\frac{\lambda}{q} \int_{\Omega}|u|^{q} \ln |u|^{2} d x+\frac{b \alpha^{2} \beta^{2}}{2} \liminf _{n \rightarrow \infty}\left(\left\|u_{n}^{+}\right\|^{2}\left\|u_{n}^{-}\right\|^{2}\right) \\
& \geq I_{b}^{\lambda}\left(\alpha u^{+}+\beta u^{-}\right)+\frac{a \alpha^{p}}{p} \lim _{n \rightarrow \infty}\left\|u_{n}^{+}-u^{+}\right\|^{p}+\frac{a \beta^{p}}{p} \lim _{n \rightarrow \infty}\left\|u_{n}^{-}-u^{-}\right\|^{p} \\
& +\frac{b \alpha^{2 p}}{p} \lim _{n \rightarrow \infty}\left\|u_{n}^{+}-u^{+}\right\|^{p}\left\|u^{+}\right\|^{p}+\frac{b \beta^{2 p}}{p} \lim _{n \rightarrow \infty}\left\|u_{n}^{-}-u^{-}\right\|^{p}\left\|u^{-}\right\|^{p} \\
& +\frac{b \alpha^{2 p}}{2 p}\left(\lim _{n \rightarrow \infty}\left\|u_{n}^{+}-u^{+}\right\|^{p}\right)^{2}+\frac{b t^{2 p}}{2 p}\left(\lim _{n \rightarrow \infty}\left\|u_{n}^{-}-u^{-}\right\|^{p}\right)^{2} \\
& -\frac{\alpha^{p_{s}^{*}}}{p_{s}^{*}} \int_{\Omega}\left|u_{n}^{+}-u^{+}\right|^{p_{s}^{*}} d x-\frac{\beta_{s}^{p_{s}^{*}}}{p_{s}^{*}} \int_{\Omega}\left|u_{n}^{-}-u^{-}\right|_{s}^{p_{s}^{*}} d x \\
& \geq I_{b}^{\lambda}\left(\alpha u^{+}+\beta u^{-}\right)+\frac{a \alpha^{p}}{p} A_{1}+\frac{b \alpha^{2 p}}{p} A_{1}\left\|u^{+}\right\|^{p}+\frac{b \alpha^{2 p}}{2 p} A_{1}^{p}-\frac{a \alpha^{p_{s}^{*}}}{p_{s}^{*}} B_{1} \\
& +\frac{a \beta^{p}}{p} A_{2}+\frac{b \beta^{2 p}}{p} A_{2}\left\|u^{-}\right\|^{p}+\frac{b \beta^{2 p}}{2 p} A_{2}^{p}-\frac{\beta^{p_{s}^{*}}}{p_{s}^{*}} B_{2},
\end{aligned}
$$

where

$$
\begin{aligned}
& A_{1}=\lim _{n \rightarrow \infty}\left\|u_{n}^{+}-u^{+}\right\|^{p}, \quad A_{2}=\lim _{n \rightarrow \infty}\left\|u_{n}^{-}-u^{-}\right\|^{p}, \\
& B_{1}=\lim _{n \rightarrow \infty}\left|u_{n}^{+}-u^{+}\right|_{p_{s}^{*}}^{p_{s}^{*}}, \quad B_{2}=\lim _{n \rightarrow \infty}\left|u_{n}^{-}-u^{-}\right|_{p_{s}^{*}}^{p_{s}^{*}}
\end{aligned}
$$

That is, one has that

$$
\begin{aligned}
& I_{b}^{\lambda}\left(\alpha u^{+}+\beta u^{-}\right)+\frac{a \alpha^{p}}{p} A_{1}+\frac{b \alpha^{2 p}}{p} A_{1}\left\|u^{+}\right\|^{p}+\frac{b \alpha^{2 p}}{2 p} A_{1}^{p}-\frac{\alpha^{p_{s}^{*}}}{p_{s}^{*}} B_{1} \\
& +\frac{a \beta^{p}}{p} A_{2}+\frac{b \beta^{2 p}}{p} A_{2}\left\|u^{-}\right\|^{p}+\frac{b \beta^{2 p}}{2 p} A_{2}^{p}-\frac{\beta^{p_{s}^{*}}}{p_{s}^{*}} B_{2} \leq c_{b}^{\lambda}
\end{aligned}
$$

for all $\alpha \geq 0$ and all $\beta \geq 0$.

Now, we claim that $u^{ \pm} \neq 0$.

In fact, since the situation $u^{-} \neq 0$ is analogous, we just prove $u^{+} \neq 0$. By contradiction, we suppose $u^{+}=0$. Hence, let $\beta=0$ in (3.17) and we have that

$$
\frac{a \alpha^{p}}{p} A_{1}+\frac{b \alpha^{2 p}}{2 p} A_{1}^{2}-\frac{\alpha^{p_{s}^{*}}}{p_{s}^{*}} B_{1} \leq c_{b}^{\lambda}
$$

for all $\alpha \geq 0$.

Case 1: $B_{1}=0$. 
If $A_{1}=0$, that is, $u_{n}^{+} \rightarrow u^{+}$in $W_{0}^{s, p}(\Omega)$. In view of Lemma (3.22), we obtain $\left\|u^{+}\right\|>0$, which contradicts our supposition. If $A_{1}>0$, by (3.22), we have that

$$
\frac{a \alpha^{p}}{p} A_{1}+\frac{b \alpha^{2 p}}{2 p} A_{1}^{2} \leq c_{b}^{\lambda}
$$

for all $\alpha \geq 0$, which is absurd by Lemma 3.3. Anyway, we have a contradiction.

Case 2: $B_{1}>0$.

One one hand, by Lemma 3.3, there exists $\lambda^{*}>0$ such that

$$
c_{b}^{\lambda}<\frac{s}{N}(a S)^{N / p s} \text { for all } \lambda \geq \lambda^{*},
$$

where $S>0$ is given by (2.2).

On the other hand, since $B_{1}>0$, we obtain $A_{1}>0$. Hence, in view of (3.22), we have that

$$
\frac{s}{N}(a S)^{N / p s} \leq \max _{\alpha \geq 0}\left\{\frac{a \alpha^{p}}{p} A_{1}-\frac{\alpha^{p_{s}^{*}}}{p_{s}^{*}} B_{1}\right\} \leq \max _{\alpha \geq 0}\left\{\frac{a \alpha^{p}}{p} A_{1}+\frac{b \alpha^{2 p}}{2 p} A_{1}^{2}-\frac{\alpha^{p_{s}^{*}}}{p_{s}^{*}} B_{1}\right\} \leq c_{b}^{\lambda},
$$

which is a contradiction. That is, we deduce that $u^{ \pm} \neq 0$.

Second, we prove $B_{1}=B_{2}=0$.

Since the situation $B_{2}=0$ is analogous, we only prove $B_{1}=0$. By contradiction, we suppose that $B_{1}>0$.

Case 1: $B_{2}>0$.

According to $B_{1}, B_{2}>0$ and Sobolev embedding, we obtain that $A_{1}, A_{2}>0$. Let

$$
\phi(\alpha)=\frac{a \alpha^{p}}{p} A_{1}+\frac{b \alpha^{2 p}}{2 p} A_{1}^{2}-\frac{\alpha^{p_{s}^{*}}}{p_{s}^{*}} B_{1} \text { for all } \alpha \geq 0 .
$$

It is easy to see that $\phi(\alpha)>0$ for $\alpha>0$ small enough and $\phi(\alpha)<0$ for $\alpha<0$ large enough. Hence, by continuous of $\phi(\alpha)$, there exists $\hat{\alpha}>0$ such that

$$
\frac{a \hat{\alpha}^{p}}{p} A_{1}+\frac{b \hat{\alpha}^{2 p}}{2 p} A_{1}^{2}-\frac{\hat{\alpha}^{p_{s}^{*}}}{p_{s}^{*}} B_{1}=\max _{\alpha \geq 0}\left\{\frac{a \alpha^{p}}{p} A_{1}+\frac{b \alpha^{2 p}}{2 p} A_{1}^{2}-\frac{\alpha^{p_{s}^{*}}}{p_{s}^{*}} B_{1}\right\} .
$$

Similarly, there exists $\hat{\beta}>0$ such that

$$
\frac{a \hat{\beta}^{p}}{p} A_{2}+\frac{b \hat{\beta}^{2 p}}{2 p} A_{2}^{2}-\frac{\hat{\beta}^{p_{s}^{*}}}{p_{s}^{*}} B_{2}=\max _{\alpha \geq 0}\left\{\frac{a \alpha^{p}}{p} B_{1}+\frac{b \alpha^{2 p}}{2 p} B_{1}^{2}-\frac{\alpha^{p_{s}^{*}}}{p_{s}^{*}} B_{1}\right\} .
$$

Since $[0, \hat{\alpha}] \times[0, \hat{\beta}]$ is compact and $\phi$ is continuous, there exists $\left(\alpha_{u}, \beta_{u}\right) \in[0, \hat{\alpha}] \times$ $[0, \hat{\beta}]$ such that

$$
\phi\left(\alpha_{u}, \beta_{u}\right)=\max _{(\alpha, \beta) \in[0, \hat{\alpha}] \times[0, \hat{\beta}]} \phi(\alpha, \beta) .
$$


Now, we prove that $\left(\alpha_{u}, \beta_{u}\right) \in(0, \hat{\alpha}) \times(0, \hat{\beta})$.

Note that, if $\beta$ is small enough, we have that

$$
\phi(\alpha, 0)=I_{b}^{\lambda}\left(\alpha u^{+}\right)<I_{b}^{\lambda}\left(\alpha u^{+}\right)+I_{b}^{\lambda}\left(\beta u^{-}\right) \leq I_{b}^{\lambda}\left(\alpha u^{+}+\beta u^{-}\right)=\phi(\alpha, \beta)
$$

for all $\alpha \in[0, \hat{\alpha}]$.

Hence, there exists $\beta_{0} \in[0, \hat{\beta}]$ such that

$$
\phi(\alpha, 0) \leq \phi\left(\alpha, \beta_{0}\right) \text { for all } \alpha \in[0, \hat{\alpha}]
$$

That is, any point of $(\alpha, 0)$ with $0 \leq \alpha \leq \hat{\alpha}$ is not the maximizer of $\phi$. Hence, $\left(\alpha_{u}, \beta_{u}\right) \notin[0, \hat{\alpha}] \times\{0\}$. Similarly, we obtain $\left(\alpha_{u}, \beta_{u}\right) \notin\{0\} \times[0, \hat{\alpha}]$.

On the other hand, it is easy to see that

$$
\frac{a \alpha^{p}}{p} A_{1}+\frac{b \alpha^{2 p}}{p} A_{1}\left\|u^{+}\right\|^{p}+\frac{b \alpha^{2 p}}{2 p} A_{1}^{2}-\frac{\alpha^{p_{s}^{*}}}{p_{s}^{*}} B_{1}>0
$$

and

$$
\frac{a \beta^{p}}{p} A_{2}+\frac{b \beta^{2 p}}{p} A_{2}\left\|u^{-}\right\|^{p}+\frac{b \beta^{2 p}}{2 p} A_{2}^{2}-\frac{\beta^{p_{s}^{*}}}{p_{s}^{*}} B_{2}>0
$$

for $\alpha \in(0, \hat{\alpha}], \beta \in(0, \hat{\beta}]$.

Then, we have that

$$
\begin{aligned}
\frac{s}{N}(a S)^{N / p s} \leq & \frac{a \hat{\alpha}^{p}}{p} A_{1}+\frac{b \hat{\alpha}^{2 p}}{2 p} A_{1}^{2}-\frac{\hat{\alpha}^{p_{s}^{*}}}{p_{s}^{*}} B_{1}+\frac{b \hat{\alpha}^{2 p}}{p} A_{1}\left\|u^{+}\right\|^{p} \\
& +\frac{a \beta^{p}}{p} A_{2}+\frac{b \beta^{2 p}}{p} A_{2}\left\|u^{-}\right\|^{p}+\frac{b \beta^{2 p}}{2 p} A_{2}^{2}-\frac{\beta^{p_{s}^{*}}}{p_{s}^{*}} B_{2}
\end{aligned}
$$

and

$$
\begin{aligned}
\frac{s}{N}(a S)^{N / p s} \leq & \frac{a \tilde{\beta}^{p}}{p} A_{2}+\frac{b \tilde{\beta}^{2 p}}{2 p} A_{2}^{p}-\frac{\tilde{\beta}^{p_{s}^{*}}}{p_{s}^{*}} B_{2}+\frac{b \tilde{\beta}^{2 p}}{p} A_{2}\left\|u^{-}\right\|^{p} \\
& +\frac{a \alpha^{p}}{p} A_{1}+\frac{b \alpha^{2 p}}{p} A_{1}\left\|u^{+}\right\|^{p}+\frac{b \alpha^{2 p}}{2 p} A_{1}^{2}-\frac{\alpha^{p_{s}^{*}}}{p_{s}^{*}} B_{1}
\end{aligned}
$$

for all $\alpha \in[0, \hat{\alpha}]$ and all $\beta \in[0, \hat{\beta}]$.

Therefore, according to (3.21), we conclude that

$$
\psi(\alpha, \hat{\beta}) \leq 0, \quad \psi(\hat{\alpha}, \beta) \leq 0
$$

for all $\alpha \in[0, \hat{\alpha}]$ and all $\beta \in[0, \hat{\beta}]$.

Hence, $\left(\alpha_{u}, \beta_{u}\right) \notin\{\hat{\alpha}\} \times[0, \hat{\beta}]$ and $\left(\alpha_{u}, \beta_{u}\right) \notin[0, \hat{\alpha}] \times\{\hat{\beta}\}$. 
Finally, we get that $\left(\alpha_{u}, \beta_{u}\right) \in(0, \hat{\alpha}) \times(0, \hat{\beta})$. Hence, it follows that $\left(\alpha_{u}, \beta_{u}\right)$ is a critical point of $\psi$.

Hence, $\alpha_{u} u^{+}+\beta_{u} u^{-} \in \mathcal{M}_{b}^{\lambda}$. From (3.17), (3.20), and (3.21), we have that

$$
\begin{aligned}
c_{b}^{\lambda} \geq & I_{b}^{\lambda}\left(\alpha_{u} u^{+}+\beta_{u} u^{-}\right)+\frac{a \alpha_{u}^{p}}{p} A_{1}+\frac{b \alpha_{u}^{2 p}}{p} A_{1}\left\|u^{+}\right\|^{p}+\frac{b \alpha_{u}^{2 p}}{2 p} A_{1}^{2}-\frac{\alpha^{p_{s}^{*}}}{p_{s}^{*}} B_{1} \\
& +\frac{a \beta_{u}^{p}}{p} A_{2}+\frac{b \beta_{u}^{2 p}}{p} A_{2}\left\|u^{-}\right\|^{p}+\frac{b \beta_{u}^{2 p}}{2 p} A_{2}^{p}-\frac{\beta^{p_{s}^{*}}}{p_{s}^{*}} B_{2} \\
> & I_{b}^{\lambda}\left(\alpha_{u} u^{+}+\beta_{u} u^{-}\right) \geq c_{b}^{\lambda},
\end{aligned}
$$

which is a contradiction.

Case 2: $B_{2}=0$.

In this case, we can maximize in $[0, \hat{\alpha}] \times[0, \infty)$. Indeed, it is possible to show that there exist $\beta_{0} \in[0, \infty)$ such that

$$
I_{b}^{\lambda}\left(\alpha_{u} u^{+}+\beta_{u} u^{-}\right) \leq 0 \text { for all }(\alpha, \beta) \in[0, \hat{\alpha}] \times\left[\beta_{0}, \infty\right) .
$$

Hence, there is $\left(\alpha_{u}, \beta_{u}\right) \in[0, \hat{\alpha}] \times[0, \infty)$ such that

$$
\phi\left(\alpha_{u}, \beta_{u}\right)=\max _{(\alpha, \beta) \in[0, \hat{\alpha}] \times[0, \infty)} \phi(\alpha, \beta) .
$$

In the following, we prove that $\left(\alpha_{u}, \beta_{u}\right) \in(0, \hat{\alpha}) \times(0, \infty)$.

It is noted that $\phi(\alpha, 0)<\phi(\alpha, \beta)$ for $\alpha \in[0, \hat{\alpha}]$ and $\beta$ small enough, so we have $\left(\alpha_{u}, \beta_{u}\right) \notin[0, \hat{\alpha}] \times\{0\}$.

Meanwhile, $\phi(0, \beta)<\phi(\alpha, \beta)$ for $\beta \in[0, \infty)$ and $\alpha$ small enough, then we have $\left(\alpha_{u}, \beta_{u}\right) \notin\{0\} \times[0, \infty)$.

On the other hand, it is obvious that

$$
\begin{aligned}
\frac{s}{N}(a S)^{N / p s} \leq & \frac{a \hat{\alpha}^{p}}{p} A_{1}+\frac{b \hat{\alpha}^{2 p}}{2 p} A_{1}^{2}-\frac{\alpha^{p_{s}^{*}}}{p_{s}^{*}} B_{1}+\frac{b \hat{\alpha}^{2 p}}{p} A_{2}\left\|u^{+}\right\|^{p} \\
& +\frac{a \beta^{p}}{p} A_{2}+\frac{b \beta^{2 p}}{p} A_{2}\left\|u^{-}\right\|^{p}+\frac{b \beta^{2 p}}{2 p} A_{2}^{2}
\end{aligned}
$$

for all $\beta \in[0, \infty)$.

Hence, we have that $\phi(\hat{\alpha}, \beta) \leq 0$ for all $\beta \in[0, \infty)$. Thus, $\left(\alpha_{u}, \beta_{u}\right) \notin\{\hat{\alpha}\} \times[0, \infty)$. Hence, $\left(\alpha_{u}, \beta_{u}\right) \in(0, \hat{\alpha}) \times(0, \infty)$. That is, $\left(\alpha_{u}, \beta_{u}\right)$ is an inner maximizer of $\phi$ in $[0, \hat{\alpha}) \times[0, \infty)$. Hence, $\alpha_{u} u^{+}+\beta_{u} u^{-} \in \mathcal{M}_{b}^{\lambda}$. 
Hence, in view of (3.24), we have that

$$
\begin{aligned}
c_{b}^{\lambda} \geq & I_{b}^{\lambda}\left(\alpha_{u} u^{+}+\beta_{u} u^{-}\right)+\frac{a \alpha_{u}^{p}}{p} A_{1}+\frac{b \alpha_{u}^{2 p}}{p} A_{1}\left\|u^{+}\right\|^{p}+\frac{b \alpha_{u}^{2 p}}{2 p} A_{1}^{2}-\frac{\alpha^{p_{s}^{*}}}{p_{s}^{*}} B_{1} \\
& +\frac{a \beta_{u}^{p}}{p} A_{2}+\frac{b \beta_{u}^{2 p}}{p} A_{2}\left\|u^{-}\right\|^{p}+\frac{b \beta_{u}^{2 p}}{2 p} A_{2}^{2} \\
> & I_{b}^{\lambda}\left(\alpha_{u} u^{+}+\beta_{u} u^{-}\right) \geq c_{b}^{\lambda},
\end{aligned}
$$

which is a contradiction.

Therefore, from the above arguments, we have that $B_{1}=B_{2}=0$.

Finally, we prove that $c_{b}^{\lambda}$ is achieved.

Since $u^{ \pm} \neq 0$, by Lemma 3.1, there exist $\alpha_{u}, \beta_{u}>0$ such that

$$
\bar{u}:=\alpha_{u} u^{+}+\beta_{u} u^{-} \in \mathcal{M}_{b}^{\lambda}
$$

Furthermore, it is easy to see that

$$
\left\langle\left(I_{b}^{\lambda}\right)^{\prime}(u), u^{ \pm}\right\rangle \leq 0
$$

By Lemma 3.2, we obtain $0<\alpha_{u}, \beta_{u} \leq 1$.

Since $u_{n} \in \mathcal{M}_{b}^{\lambda}$, according to Lemma 3.3, we get

$$
I_{b}^{\lambda}\left(\alpha_{u} u_{n}^{+}+\beta_{u} u_{n}^{-}\right) \leq I_{b}^{\lambda}\left(u_{n}^{+}+u_{n}^{-}\right)=I_{b}^{\lambda}\left(u_{n}\right)
$$

Thanks to $B_{1}=B_{2}=0$ and the norm in $W_{0}^{s, p}(\Omega)$ is lower semicontinuous, and we have that

$$
\begin{aligned}
c_{b}^{\lambda} \leq & I_{b}^{\lambda}(\bar{u})-\frac{1}{q}\left\langle\left(I_{b}^{\lambda}\right)^{\prime}(\bar{u}), \bar{u}\right\rangle \\
\leq & \left(\frac{1}{p}-\frac{1}{q}\right) a\|\bar{u}\|^{p}+\left(\frac{1}{2 p}-\frac{1}{q}\right) b\|\bar{u}\|^{2 p}+\frac{2 \lambda}{q^{2}} \int_{\Omega}|\bar{u}|^{q} d x+\left(\frac{1}{q}-\frac{1}{p_{s}^{*}}\right) \int_{\Omega}|\bar{u}|^{p_{s}^{*}} d x \\
= & \left(\frac{1}{p}-\frac{1}{q}\right) a\left(\left\|\alpha_{u} u^{+}\right\|^{p}+\left\|\beta_{u} u^{-}\right\|^{p}\right)+\left(\frac{1}{2 p}-\frac{1}{q}\right) b\left(\left\|\alpha_{u} u^{+}\right\|^{p}+\left\|\beta_{u} u^{-}\right\|^{p}\right)^{2} \\
& +\frac{2 \lambda}{q^{2}}\left[\int_{\Omega}\left|\alpha_{u} u^{+}\right|^{q} d x+\int_{\Omega}\left|\beta_{u} u^{-}\right|^{q} d x\right]+\left(\frac{1}{q}-\frac{1}{p_{s}^{*}}\right)\left[\int_{\Omega}\left|\alpha_{u} u^{+}\right|^{p_{s}^{*}} d x\right. \\
& \left.+\int_{\Omega}\left|\beta_{u} u^{-}\right|^{p_{s}^{*}} d x\right] \\
\leq & \left(\frac{1}{p}-\frac{1}{q}\right) a\|u\|^{p}+\left(\frac{1}{2 p}-\frac{1}{q}\right) b\|u\|^{2 p}+\frac{2 \lambda}{q^{2}} \int_{\Omega}|u|^{q} d x+\left(\frac{1}{q}-\frac{1}{p_{s}^{*}}\right) \int_{\Omega}|u|^{p_{s}^{*}} d x \\
\leq & \liminf _{n \rightarrow \infty}\left[I_{b}^{\lambda}\left(u_{n}\right)-\frac{1}{q}\left\langle\left(I_{b}^{\lambda}\right)^{\prime}\left(u_{n}\right), u_{n}\right\rangle\right] \leq c_{b}^{\lambda} .
\end{aligned}
$$

Therefore, $\alpha_{u}=\beta_{u}=1$, and $c_{b}^{\lambda}$ is achieved by $u_{b}:=u^{+}+u^{-} \in \mathcal{M}_{b}^{\lambda}$. 


\section{Proof of Theorems}

In this section, we prove our main results. First, we prove Theorem 2.1. In fact, thanks to Lemma 3.4, we just prove that the minimizer $u_{b}$ for $c_{b}^{\lambda}$ is indeed a sign-changing solution of problem (1.1).

Proof of Theorem 2.1 Since $u_{b} \in \mathcal{M}_{b}^{\lambda}$, we have $\left\langle\left(I_{b}^{\lambda}\right)^{\prime}\left(u_{b}\right), u_{b}^{+}\right\rangle=\left\langle\left(I_{b}^{\lambda}\right)^{\prime}\left(u_{b}\right), u_{b}^{-}\right\rangle=$ 0 . By Lemma 3.4, for $(\alpha, \beta) \in\left(\mathbb{R}_{+} \times \mathbb{R}_{+}\right) \backslash(1,1)$, we have

$$
I_{b}^{\lambda}\left(\alpha u_{b}^{+}+\beta u_{b}^{-}\right)<I_{b}^{\lambda}\left(u_{b}^{+}+u_{b}^{-}\right)=c_{b}^{\lambda} .
$$

Arguing by contradiction, we assume that $\left(I_{b}^{\lambda}\right)^{\prime}\left(u_{b}\right) \neq 0$, then there exist $\delta>0$ and $\iota>0$ such that

$$
\left\|\left(I_{b}^{\lambda}\right)^{\prime}(v)\right\| \geq \iota \text { for all }\left\|v-u_{b}\right\| \geq 3 \delta
$$

Choose $\tau \in\left(0, \min \left\{1 / 2, \frac{\delta}{\sqrt{2}\left\|u_{b}\right\|}\right\}\right)$. Let

$$
D:=(1-\tau, 1+\tau) \times(1-\tau, 1+\tau)
$$

and

$$
g(\alpha, \beta)=\alpha u_{b}^{+}+\beta u_{b}^{-} \text {for all }(\alpha, \beta) \in D
$$

In view of (4.1), it is easy to see that

$$
\bar{c}_{\lambda}:=\max _{\partial \Omega} I_{b}^{\lambda} \circ g<c_{b, \lambda} .
$$

Let $\varepsilon:=\min \left\{\left(c_{b}^{\lambda}-\bar{c}_{\lambda}\right) / 3, \iota \delta / 8\right\}$ and $S_{\delta}:=B\left(u_{b}, \delta\right)$, according to Lemma 2.3 in [46], there exists a deformation $\eta \in C([0,1] \times D, D)$ such that

(a) $\eta(1, v)=v$ if $v \notin\left(I_{b}^{\lambda}\right)^{-1}\left(\left[c_{b}^{\lambda}-2 \varepsilon, c_{b}^{\lambda}+2 \varepsilon\right] \cap S_{2 \delta}\right)$,

(b) $\eta\left(1,\left(I_{b}^{\lambda}\right)^{c_{b}^{\lambda}+\varepsilon} \cap S_{\delta}\right) \subset\left(I_{b}^{\lambda}\right)^{c_{b, \lambda}-\varepsilon}$,

(c) $\left.I_{b}^{\lambda}(\eta(1, v)) \leq I_{b}^{\lambda}\right)(v)$ for all $v \in W_{0}^{s, p}(\Omega)$.

First, from (b) and Lemma 3.2, it is easy to see that

$$
\max _{(\alpha, \beta) \in \bar{D}} I_{b}^{\lambda}(\eta(1, g(\alpha, \beta)))<c_{b}^{\lambda}
$$

Next, we prove that $\eta(1, g(D)) \cap \mathcal{M}_{b}^{\lambda} \neq \varnothing$, which contradicts the definition of $c_{b}^{\lambda}$. Let $\gamma(\alpha, \beta):=\eta(1, g(\alpha, \beta))$ and

$$
\begin{aligned}
\Psi_{0}(\alpha, \beta) & :=\left(\left\langle\left(I_{b}^{\lambda}\right)^{\prime}(g(\alpha, \beta)), u_{b}^{+}\right\rangle,\left\langle\left(I_{b}^{\lambda}\right)^{\prime}(g(\alpha, \beta)), u_{b}^{-}\right\rangle\right) \\
& =\left(\left\langle\left(I_{b}^{\lambda}\right)^{\prime}\left(\alpha u_{b}^{+}+\beta u_{b}^{-}\right), u_{b}^{+}\right\rangle,\left\langle\left(I_{b}^{\lambda}\right)^{\prime}\left(\alpha u_{b}^{+}+\beta u_{b}^{-}\right), u_{b}^{-}\right\rangle\right) \\
& :=\left(\varphi_{u}^{1}(\alpha, \beta), \varphi_{u}^{2}(\alpha, \beta)\right)
\end{aligned}
$$


and

$$
\Psi_{1}(\alpha, \beta):=\left(\frac{1}{\alpha}\left\langle\left(I_{b}^{\lambda}\right)^{\prime}(\gamma(\alpha, \beta)),(\gamma(\alpha, \beta))^{+}\right\rangle, \frac{1}{\beta}\left\langle\left(I_{b}^{\lambda}\right)^{\prime}(\gamma(\alpha, \beta)),(\gamma(\alpha, \beta))^{-}\right\rangle\right) .
$$

Since $u_{b} \in \mathcal{M}_{b}^{\lambda}$, by the direct calculation, we have

$$
\begin{aligned}
\left.\frac{\varphi_{u}^{1}(\alpha, \beta)}{\partial \alpha}\right|_{(1,1)}= & a(p-1)\left\|u_{b}^{+}\right\|^{p}+b(2 p-1)\left\|u_{b}^{+}\right\|^{2 p}+b(p-1)\left\|u_{b}^{+}\right\|^{p}\left\|u_{b}^{-}\right\|^{p} \\
& -\lambda(q-1) \int_{\Omega}\left|u_{b}^{+}\right|^{q} \ln \left|u_{b}^{+}\right|^{2} d x-2 \int_{\Omega}\left|u_{b}^{+}\right|^{q} d x-\left(p_{s}^{*}-1\right) \int_{\Omega}\left|u_{b}^{+}\right|^{p_{s}^{*}} d x \\
= & b p\left\|u_{b}^{+}\right\|^{2 p}-\lambda(q-p) \int_{\Omega}\left|u_{b}^{+}\right|^{q} \ln \left|u_{b}^{+}\right|^{2} d x-2 \int_{\Omega}\left|u_{b}^{+}\right|^{q} d x \\
& -\left(p_{s}^{*}-p\right) \int_{\Omega}\left|u_{b}^{+}\right|^{p_{s}^{*}} d x
\end{aligned}
$$

and

$$
\left.\frac{\varphi_{u}^{1}(\alpha, \beta)}{\partial \beta}\right|_{(1,1)}=p b\left\|u_{b}^{+}\right\|^{p}\left\|u_{b}^{-}\right\|^{p} .
$$

Similarly, we have

$$
\begin{aligned}
\left.\frac{\varphi_{u}^{2}(\alpha, \beta)}{\partial \beta}\right|_{(1,1)}= & b p\left\|u_{b}^{-}\right\|^{2 p}-\lambda(q-p) \int_{\Omega}\left|u_{b}^{-}\right|^{q} \ln \left|u_{b}^{-}\right|^{2} d x-2 \int_{\Omega}\left|u_{b}^{-}\right|^{q} d x \\
& -\left(p_{s}^{*}-p\right) \int_{\Omega}\left|u_{b}^{-}\right|^{p_{s}^{*}} d x
\end{aligned}
$$

and

$$
\left.\frac{\varphi_{u}^{2}(\alpha, \beta)}{\partial \alpha}\right|_{(1,1)}=p b\left\|u_{b}^{+}\right\|^{p}\left\|u_{b}^{-}\right\|^{p}
$$

Let

$$
M=\left[\begin{array}{ll}
\left.\left.\frac{\varphi_{u}^{1}(\alpha, \beta)}{\partial \alpha}\right|_{(1,1)} \frac{\varphi_{u}^{2}(\alpha, \beta)}{\partial \alpha}\right|_{(1,1)} \\
\left.\left.\frac{\varphi_{u}^{1}(\alpha, \beta)}{\partial \beta}\right|_{(1,1)} \frac{\varphi_{u}^{2}(\alpha, \beta)}{\partial \beta}\right|_{(1,1)}
\end{array}\right]
$$

Then, we have that

$$
\operatorname{det} M=\left.\frac{\varphi_{u}^{1}(\alpha, \beta)}{\partial \alpha}\right|_{(1,1)} \times\left.\frac{\varphi_{u}^{2}(\alpha, \beta)}{\partial \beta}\right|_{(1,1)}-\left.\frac{\varphi_{u}^{1}(\alpha, \beta)}{\partial \beta}\right|_{(1,1)} \times\left.\frac{\varphi_{u}^{2}(\alpha, \beta)}{\partial \alpha}\right|_{(1,1) \neq 0 .}
$$

Since $\Psi_{0}(\alpha, \beta)$ is a $C^{1}$ function and $(1,1)$ is the unique isolated zero point of $\Psi_{0}$, by using the degree theory, we deduce that $\operatorname{deg}\left(\Psi_{0}, D, 0\right)=1$. 
Hence, combining (4.3) with (a), we obtain

$$
g(\alpha, \beta)=\gamma(\alpha, \beta) \text { on } \partial D
$$

Consequently, we obtain $\operatorname{deg}\left(\Psi_{1}, D, 0\right)=1$. Therefore, $\Psi_{1}\left(\alpha_{0}, \beta_{0}\right)=0$ for some $\left(\alpha_{0}, \beta_{0}\right) \in D$ so that

$$
\eta\left(1, g\left(\alpha_{0}, \beta_{0}\right)\right)=\gamma\left(\alpha_{0}, \beta_{0}\right) \in \mathcal{M}_{b}^{\lambda}
$$

which is contradicted to (4.3).

From the above discussions, we deduce that $u_{b}$ is a sign-changing solution for problem (1.1).

Finally, we prove that u has exactly two nodal domains. To this end, we assume by contradiction that

$$
u_{b}=u_{1}+u_{2}+u_{3}
$$

where

$$
\begin{aligned}
& u_{i} \neq 0, u_{1} \geq 0, u_{2} \leq 0, \Omega_{1} \cap \Omega_{2}=\emptyset, u_{1}\left|\Omega_{\Omega} \Omega_{1} \cup \Omega_{2}=u_{2}\right| \Omega \backslash \Omega_{1} \cup \Omega_{2}=u_{3} \mid \Omega_{1} \cap \Omega_{2}=0, \\
& \Omega_{1}:=\left\{x \in \Omega: u_{1}(x)>0\right\} \text { and } \Omega_{2}:=\left\{x \in \Omega: u_{2}(x)<0\right\} \text { for } i \neq j, i, j=1,2,3
\end{aligned}
$$

are two connected open subsets of $\Omega$, and

$$
\left\langle\left(I_{b}^{\lambda}\right)^{\prime}(u), u_{i}\right\rangle=0 \text { for } i=1,2,3 \text {. }
$$

Setting $v:=u_{1}+u_{2}$, we see that $v^{+}=u_{1}$ and $v^{-}=u_{2}$, i.e., $v^{ \pm} \neq 0$. Then, there exist a unique pair $\left(\alpha_{v}, \beta_{v}\right)$ of positive numbers such that

$$
\alpha_{v} u_{1}+\beta_{v} u_{2} \in \mathcal{M}_{b}^{\lambda}
$$

Hence

$$
I_{b}^{\lambda}\left(\alpha_{v} u_{1}+\beta_{v} u_{2}\right) \geq c_{b}^{\lambda}
$$

Moreover, using the fact that $\left\langle\left(I_{b}^{\lambda}\right)^{\prime}(u), u_{i}\right\rangle=0$, we obtain $\left\langle\left(I_{b}^{\lambda}\right)^{\prime}(v), v^{ \pm}\right\rangle=$ $-b\left\|v^{ \pm}\right\|{ }^{p}\left\|u_{3}\right\|^{p}<0$.

From Lemma 3.1 (ii), we have that

$$
\left(\alpha_{v}, \beta_{v}\right) \in(0,1] \times(0,1] .
$$


On the other hand, we have that

$$
\begin{aligned}
0=\frac{1}{2 p}\left\langle\left(I_{b}^{\lambda}\right)^{\prime}(u), u_{3}\right\rangle= & \frac{1}{2 p} a\left\|u_{3}\right\|^{p}+\frac{b}{2 p}\left\|u_{1}\right\|^{p}\left\|u_{3}\right\|^{p}+\frac{b}{2 p}\left\|u_{2}\right\|^{p}\left\|u_{3}\right\|^{p}+\frac{b}{2 p}\left\|u_{3}\right\|^{2 p} \\
& -\frac{1}{2 p} \int_{\Omega}\left|u_{3}\right|^{p_{s}^{*}} d x-\frac{\lambda}{2 p} \int_{\Omega}\left|u_{3}^{+}\right|^{q} \ln \left|u_{3}^{+}\right|^{2} d x \\
& <I_{b}^{\lambda}\left(u_{3}\right)+\frac{b}{2 p}\left\|u_{1}\right\|^{2}\left\|u_{3}\right\|^{p}+\frac{b}{2 p}\left\|u_{2}\right\|^{2}\left\|u_{3}\right\|^{p}
\end{aligned}
$$

Hence, by (3.15), we can obtain that

$$
\begin{aligned}
c_{b}^{\lambda} \leq & I_{b}^{\lambda}\left(\alpha_{v} u_{1}+\beta_{v} u_{2}\right)=I_{b}^{\lambda}\left(\alpha_{v} u_{1}+\beta_{v} u_{2}\right)-\frac{1}{2 p}\left\langle\left(I_{b}^{\lambda}\right)^{\prime}\left(\alpha_{v} u_{1}+\beta_{v} u_{2}\right),\left(\alpha_{v} u_{1}+\beta_{v} u_{2}\right)\right\rangle \\
= & \frac{a}{2 p}\left(\left\|\alpha_{v} u_{1}\right\|^{p}+\left\|\beta_{v} u_{2}\right\|^{p}\right)+\frac{\lambda}{q^{2}}\left[\int_{\Omega}\left|\alpha_{v} u_{1}\right|^{q} d x+\int_{\Omega}\left|\beta_{v} u_{2}\right|^{q} d x\right] \\
& +\left(\frac{1}{2 p}-\frac{1}{q}\right) \lambda\left[\int_{\Omega}\left[\left|\alpha_{v} u_{1}\right|^{q} \ln \left|\alpha_{v} u_{1}\right|^{2} d x+\int_{\Omega}\left|\beta_{v} u_{2}\right|^{q} \ln \left|\beta_{v} u_{2}\right|^{2} d x\right]\right. \\
& +\left(\frac{1}{2 p}-\frac{1}{p_{s}^{*}}\right) \int_{\Omega} \alpha_{v}^{p_{s}^{*}}\left|u_{1}\right|^{p_{s}^{*}} d x+\left(\frac{1}{2 p}-\frac{1}{p_{s}^{*}}\right) \int_{\Omega} \beta_{v}^{p_{s}^{*}}\left|u_{2}\right|^{p_{s}^{*}} d x \\
\leq & \frac{1}{2 p}\left(\left\|u_{1}\right\|^{p}+\left\|u_{2}\right\|^{p}\right)+\frac{\lambda}{q^{2}}\left[\int_{\Omega}\left|u_{1}\right|^{q} d x+\int_{\Omega}\left|u_{2}\right|^{q} d x\right] \\
& +\left(\frac{1}{2 p}-\frac{1}{q}\right) \lambda\left[\int_{\Omega}\left|u_{1}\right|^{q} \ln \left|u_{1}\right|^{2} d x+\int_{\Omega}\left|u_{2}\right|^{q} \ln \left|u_{2}\right|^{2} d x\right] \\
& +\left(\frac{1}{2 p}-\frac{1}{p_{s}^{*}}\right) \int_{\Omega}\left|u_{1}\right|^{p_{s}^{*}} d x+\left(\frac{1}{2 p}-\frac{1}{p_{s}^{*}}\right) \int_{\Omega}\left|u_{2}\right|^{p_{s}^{*}} d x \\
= & I_{b}^{\lambda}\left(u_{1}+u_{2}\right)-\frac{1}{2 p}\left\langle\left(I_{b}^{\lambda}\right)^{\prime}\left(u_{1}+u_{2}\right),\left(u_{1}+u_{2}\right)\right\rangle \\
= & I_{b}^{\lambda}\left(u_{1}+u_{2}\right)+\frac{1}{2 p}\left\langle\left(I_{b}^{\lambda}\right)^{\prime}(u), u_{3}\right\rangle+\frac{b}{2 p}\left\|u_{1}\right\|^{p}\left\|u_{3}\right\|^{p}+\frac{b}{2 p}\left\|u_{2}\right\|^{p}\left\|u_{3}\right\|^{p} \\
< & I_{b}^{\lambda}\left(u_{1}\right)+I_{b}^{\lambda}\left(u_{2}\right)+I_{b}^{\lambda}\left(u_{3}\right)+\frac{b}{2 p}\left(\left\|u_{2}\right\|^{p}+\left\|u_{3}\right\|^{p}\right)\left\|u_{1}\right\|^{p} \\
& +\frac{b}{2 p}\left(\left\|u_{1}\right\|^{p}+\left\|u_{3}\right\|^{p}\right)\left\|u_{2}\right\|^{p}+\frac{b}{2 p}\left(\left\|u_{1}\right\|^{p}+\left\|u_{2}\right\|^{p}\right)\left\|u_{3}\right\|^{p} \\
= & I_{b}^{\lambda}(u)=c_{b}^{\lambda},
\end{aligned}
$$

which is a contradiction, that is, $u_{3}=0$ and $u_{b}$ has exactly two nodal domains.

By Theorem 2.1, we obtain a least energy sign-changing solution $u_{b}$ of problem (1.1). Next,we prove that the energy of $u_{b}$ is strictly larger than two times the ground state energy.

Proof of Theorem 2.2 Similar to Proof of Lemma 3.3, there exists $\lambda_{1}^{*}>0$ such that for all $\lambda \geq \lambda_{1}^{*}$, and for each $b>0$, there exists $v_{b} \in \mathcal{N}_{b}^{\lambda}$ such that $I_{b}^{\lambda}\left(v_{b}\right)=c^{*}>0$. By standard arguments (see Corollary 2.13 in Ref. [22]), the critical points of the functional $I_{b}^{\lambda}$ on $\mathcal{N}_{b}^{\lambda}$ are critical points of $I_{b}^{\lambda}$ in $W_{0}^{s, p}(\Omega)$, and we obtain $\left(I_{b}^{\lambda}\right)^{\prime}\left(v_{b}\right)=0$. That is, $v_{b}$ is a ground state solution of (1.1). 
According to Theorem 2.1, we know that the problem (1.1) has a least energy sign-changing solution $u_{b}$, which changes sign only once when $\lambda \geq \lambda^{*}$.

Let

$$
\lambda^{* *}=\max \left\{\lambda^{*}, \lambda_{1}^{*}\right\}
$$

Suppose that $u_{b}=u_{b}^{+}+u_{b}^{-}$. As Proof of Lemma 3.1, there exist $\alpha_{u_{b}^{+}}>0$ and $\beta_{u_{b}^{-}}>0$ such that

$$
\alpha_{u_{b}^{+}} u_{b}^{+} \in \mathcal{N}_{b}^{\lambda}, \quad \beta_{u_{b}^{-}} u_{b}^{-} \in \mathcal{N}_{b}^{\lambda}
$$

Furthermore, Lemma 3.2 implies that $\alpha_{u_{b}^{+}}, \beta_{u_{b}^{-}} \in(0,1)$.

Therefore, in view of Lemma 3.1, we have that

$$
2 c^{*} \leq I_{b}^{\lambda}\left(\alpha_{u_{b}^{+}} u_{b}^{+}\right)+I_{b}^{\lambda}\left(\beta_{u_{b}^{-}} u_{b}^{-}\right) \leq I_{b}^{\lambda}\left(\alpha_{u_{b}^{+}} u_{b}^{+}+\beta_{u_{b}^{-}} u_{b}^{-}\right)<I_{b}^{\lambda}\left(u_{b}^{+}+u_{b}^{-}\right)=c_{b}^{\lambda} \text {. }
$$

Hence, it follows that $c^{*}>0$ cannot be achieved by a sign-changing function.

Finally, we close this section with the proof of Theorem 2.3. In the following, we regard $b>0$ as a parameter in problem (1.1).

Proof of Theorem 2.3 We shall proceed through several steps on analyzing the convergence property of $u_{b}$ as $b \rightarrow 0$, where $u_{b}$ is the least energy sign-changing solution obtained in Theorem 2.1.

Step 1 For any sequence $\left\{b_{n}\right\}$ as $b_{n} \searrow 0,\left\{u_{b_{n}}\right\}$ is bounded in $W_{0}^{s, p}(\Omega)$.

Choose a nonzero function $\omega \in C_{0}^{\infty}(\Omega)$ with $\omega^{ \pm}=0$. Similar to discussion as in Lemma 3.2, for any $\lambda \in[0,1]$, there exists a pair positive numbers $\left(\lambda_{1}, \lambda_{2}\right)$ independent of $\lambda$, such that

$$
\left\langle\left(I_{b}^{\lambda}\right)^{\prime}\left(\lambda_{1} \omega^{+}+\lambda_{2} \omega^{-}\right), \lambda_{1} \omega^{+}\right\rangle<0, \quad\left\langle\left(I_{b}^{\lambda}\right)^{\prime}\left(\lambda_{1} \omega^{+}+\lambda_{2} \omega^{-}\right), \lambda_{2} \omega^{-}\right\rangle<0 .
$$

Then by virtue of Lemma 3.1, we get that, for any $b \in[0,1]$, there exists a unique pair $\left(\alpha_{\omega}(b), \beta_{\omega}(b)\right) \in(0,1] \times(0,1]$ such that

$$
\left.\bar{\omega}:=\alpha_{\omega}(b) \lambda_{1} \omega^{+}+\beta_{\omega}(b)\right) \lambda_{2} \omega^{-} \in \mathcal{M}_{b}^{\lambda}
$$

Thus, for any $\lambda \in[0,1]$, we have

$$
\begin{aligned}
I_{b}^{\lambda}\left(u_{b}\right)= & I_{b}^{\lambda}(\bar{\omega})=I_{b}^{\lambda}(\bar{\omega})-\frac{1}{2 p}\left\langle\left(I_{b}^{\lambda}\right)^{\prime}(\bar{\omega}), \bar{\omega}\right\rangle \\
= & \frac{a}{2 p}\|\bar{\omega}\|^{p}+\left(\frac{1}{2 p}-\frac{1}{p_{s}^{*}}\right) \int_{\Omega}|\bar{\omega}|^{p_{s}^{*}} d x \\
& +\frac{\lambda}{q^{2}} \int_{\Omega}|\bar{\omega}|^{q} d x+\left(\frac{1}{2 p}-\frac{1}{q}\right) \lambda \int_{\Omega}|\bar{\omega}|^{q} \ln |\bar{\omega}|^{2} d x
\end{aligned}
$$




$$
\begin{aligned}
\leq & \frac{a}{2 p}\|\bar{\omega}\|^{p}+\left(\frac{1}{2 p}-\frac{1}{p_{s}^{*}}\right) \int_{\Omega}|\bar{\omega}|^{p_{s}^{*}} d x \\
& +\frac{\lambda}{q^{2}} \int_{\Omega}|\bar{\omega}|^{q} d x+\left(\frac{1}{2 p}-\frac{1}{q}\right) \lambda \int_{\Omega}\left(C_{1}|\bar{\omega}|^{p}+C_{2}|\bar{\omega}|^{r}\right) d x \\
\leq & \frac{a}{2 p}\left\|\lambda_{1} \omega^{+}\right\|^{p}+\frac{1}{2 p}\left\|\lambda_{2} \omega^{-}\right\|^{p}+\left(\frac{1}{2 p}-\frac{1}{p_{s}^{*}}\right) \int_{\Omega}\left|\lambda_{1} \omega^{+}\right|^{p_{s}^{*}} d x \\
& +\left(\frac{1}{2 p}-\frac{1}{p_{s}^{*}}\right) \int_{\Omega}\left|\lambda_{2} \omega^{-}\right|^{p_{s}^{*}} d x \\
& +\frac{\lambda}{q^{2}} \int_{\Omega}\left|\lambda_{1} \omega^{+}\right|^{q} d x+\frac{\lambda}{q^{2}} \int_{\Omega}\left|\lambda_{2} \omega^{-}\right|^{q} d x \\
& +\left(\frac{1}{2 p}-\frac{1}{q}\right) \lambda \int_{\Omega}\left(C_{1} \lambda_{1}^{2}\left|\omega^{+}\right|^{2}+C_{2} \lambda_{2}^{r}\left|\omega^{+}\right|^{r}\right) d x \\
& +\left(\frac{1}{2 p}-\frac{1}{q}\right) \lambda \int_{\Omega}\left(C_{1} \lambda_{1}^{2}\left|\omega^{-}\right|^{2}+C_{2} \lambda_{2}^{r}\left|\omega^{-}\right|^{r}\right) d x:=C^{*},
\end{aligned}
$$

where $C^{*}>0$ is a constant independent of $\lambda$. So, let $n \rightarrow \infty$, it follows that

$$
C^{*}+1 \geq I_{b_{n}}^{\lambda}\left(u_{b_{n}}\right)=I_{b_{n}}^{\lambda}\left(u_{b_{n}}\right)-\frac{1}{2 p}\left\langle\left(I_{b_{n}}^{\lambda}\right)^{\prime}\left(u_{b_{n}}\right), u_{b_{n}}\right\rangle \geq \frac{1}{2 p}\left\|u_{b_{n}}\right\|^{2},
$$

which implies that $\left\{u_{b_{n}}\right\}$ is bounded in $W_{0}^{s, p}(\Omega)$.

Step 2 Problem (2.12) possesses one sign-changing solution $u_{0}$.

Since $\left\{u_{b_{n}}\right\}$ is bounded in $W_{0}^{s, p}(\Omega)$, according to Step 1, going if necessary to a subsequence, there exists $u_{0} \in W_{0}^{s, p}(\Omega)$ such that

$$
\begin{aligned}
& u_{b_{n}} \rightarrow u_{0} \text { in } W_{0}^{s, p}(\Omega), \\
& u_{b_{n}} \rightarrow u_{0} \text { in } L^{t}(\Omega) \text { for } t \in\left[p, p_{s}^{*}\right), \\
& u_{b_{n}} \rightarrow u_{0} \text { a.e. } x \in \Omega .
\end{aligned}
$$

Since $\left\{u_{b_{n}}\right\}$ is a weak solution of (1.1) with $b=b_{n}$, we have

$$
\left(a+b_{n}[u]_{s, p}^{p}\right) L(u, v)=\lambda \int_{\Omega}|u|^{q-2} u v \ln |u|^{2} d x+\int_{\Omega}|u|^{p_{s}^{*}-2} u v d x
$$

for all $v \in C_{0}^{\infty}(\Omega), L(u, v)$ is defined by $(2.5)$.

From (4.5), (4.6) and Step 1, we find that

$$
\left(a+b_{n}\left[u_{0}\right]_{s, p}^{p}\right) L\left(u_{0}, v\right)=\lambda \int_{\Omega}|u|^{q-2} u_{0} v \ln \left|u_{0}\right|^{2} d x+\int_{\Omega}\left|u_{0}\right|^{p_{s}^{*}-2} u_{0} v d x
$$

for all $v \in C_{0}^{\infty}(\Omega)$, which in turn implies that $u_{0}$ is a weak solution of problem (2.12). By a similar argument as in the proof of Lemma 3.3, we conclude that $u_{0}^{ \pm} \neq 0$. Therefore, we complete the proof of the Step 2. 
Step 3 Problem (2.12) possesses a least energy sign-changing solution $v_{0}$, and there exists a unique pair $\left(\alpha_{b_{n}}, \beta_{b_{n}}\right) \in[0, \infty) \times[0, \infty)$ such that $\alpha_{b_{n}} v_{0}^{+}+\beta_{b_{n}} v_{0}^{-} \in \mathcal{M}_{b_{n}}^{\lambda}$. Moreover, $\left(\alpha_{b_{n}}, \beta_{b_{n}}\right) \rightarrow(1,1)$ as $n \rightarrow \infty$.

By a similar argument to the proof of Theorem 2.1, we have that problem (2.12) possesses a least energy sign-changing solution $v_{0}$, where $I_{0}^{\lambda}\left(v_{0}\right)=c_{0}$ nod and $\left(I_{0}^{\lambda}\right)^{\prime}\left(v_{0}\right)=0$. Then, by Lemma 3.1, we can easily obtain the existence and uniqueness of the pair $\left(\alpha_{b_{n}}, \beta_{b_{n}}\right)$ such that $\alpha_{b_{n}} v_{0}^{+}+\beta_{b_{n}} v_{0}^{-} \in \mathcal{M}_{b_{n}}^{\lambda}$. Moreover, we have $\alpha_{b_{n}}>0$ and $\beta_{b_{n}}>0$. Then the claim will follow once we can prove that $\left(\alpha_{b_{n}}, \beta_{b_{n}}\right) \rightarrow(1,1)$ as $n \rightarrow \infty$. In fact, since $\alpha_{b_{n}} v_{0}^{+}+\beta_{b_{n}} v_{0}^{-} \in \mathcal{M}_{b_{n}}^{\lambda}$, we have that

$$
\begin{gathered}
\alpha_{b_{n}}^{p} a\left\|v_{0}^{+}\right\|^{p}+b_{n} \alpha_{b_{n}}^{2 p}\left\|v_{0}^{+}\right\|^{2 p}+b_{n} \alpha_{b_{n}}^{p} \beta_{b_{n}}^{p}\left\|v_{0}^{+}\right\|^{p}\left\|v_{0}^{-}\right\|^{p} \\
=\lambda \int_{\Omega}\left|\alpha_{b_{n}} v_{0}^{+}\right|^{q} \ln \left|\alpha_{b_{n}} v_{0}^{+}\right|^{2} d x+\int_{\Omega}\left|\alpha_{b_{n}} v_{0}^{+}\right|^{p_{s}^{*}} d x
\end{gathered}
$$

and

$$
\begin{aligned}
& \beta_{b_{n}}^{p} a\left\|v_{0}^{-}\right\|^{p}+b_{n} \beta_{b_{n}}^{2 p}\left\|v_{0}^{-}\right\|^{2 p}+b_{n} \alpha_{b_{n}}^{p} \beta_{b_{n}}^{p}\left\|v_{0}^{+}\right\|^{p}\left\|v_{0}^{-}\right\|^{p} \\
& \quad=\lambda \int_{\Omega}\left|\beta_{b_{n}} v_{0}^{+}\right|^{q} \ln \left|\beta_{b_{n}} v_{0}^{+}\right|^{2} d x+\int_{\Omega}\left|\beta_{b_{n}} v_{0}^{-}\right|^{p_{s}^{*}} d x .
\end{aligned}
$$

From the convergence of $b_{n}$ as $n \rightarrow \infty$, we deduce that the sequences $\left\{\alpha_{b_{n}}\right\}$ and $\left\{\beta_{b_{n}}\right\}$ are bounded. Up to a subsequence, suppose that $\alpha_{b_{n}} \rightarrow \alpha_{0}$ and $\beta_{b_{n}} \rightarrow \beta_{0}$. Then it follows from (4.8) and (4.9) that

$$
\alpha_{0}^{p} a\left\|v_{0}^{+}\right\|^{p}=\lambda \int_{\Omega}\left|\alpha_{0} v_{0}^{+}\right|^{q} \ln \left|\alpha_{0} v_{0}^{+}\right|^{2} d x+\int_{\Omega}\left|\alpha_{0} v_{0}^{+}\right|^{2^{* *}} d x
$$

and

$$
\beta_{0}^{p} a\left\|v_{0}^{-}\right\|^{p}=\lambda \int_{\Omega}\left|\beta_{0} v_{0}^{-}\right|^{q} \ln \left|\beta_{0} v_{0}^{-}\right|^{2} d x+\int_{\Omega}\left|\beta_{0} v_{0}^{-}\right|^{2^{* *}} d x .
$$

Thanks to $v_{0}$ is a sign-changing solution of problem (2.12), we get

$$
a\left\|v_{0}^{ \pm}\right\|^{2}=\lambda \int_{\Omega}\left|v_{0}^{ \pm}\right|^{q} \ln \left|v_{0}^{ \pm}\right|^{2} d x+\int_{\Omega}\left|v_{0}^{ \pm}\right|^{p_{s}^{*}} d x .
$$

Hence, in view of (4.10)-(4.12), we can easily obtain that $\left(\alpha_{0}, \beta_{0}\right)=(1,1)$, and the Step 3 follows.

Now, we can now give the proof of Theorem 2.3. We assert that $u_{0}$ obtained in Step 2 is a least energy solution of problem (2.12). In fact, by virtue of Step 3 and Lemma 3.1, we find that

$$
\begin{aligned}
I_{0}^{\lambda}\left(v_{0}\right) \leq I_{0}^{\lambda}\left(u_{0}\right) & =\lim _{n \rightarrow \infty} I_{b_{n}}^{\lambda}\left(u_{b_{n}}\right) \leq \lim _{n \rightarrow \infty} I_{b_{n}}^{\lambda}\left(\alpha_{b_{n}} v_{0}^{+}+\beta_{b_{n}} v_{0}^{-}\right) \\
& =\lim _{n \rightarrow \infty} I_{0}^{\lambda}\left(v_{0}^{+}+v_{0}^{-}\right)=I_{0}^{\lambda}\left(v_{0}\right)
\end{aligned}
$$


Hence, the proof of Theorem 2.3 is completed.

Acknowledgements S. Liang was supported by the Foundation for China Postdoctoral Science Foundation (Grant No. 2019M662220), Natural Science Foundation of Jilin Province, Research Foundation during the 13th Five-Year Plan Period of Department of Education of Jilin Province, China (JJKH20181161KJ), Natural Science Foundation of Changchun Normal University (No. 2017-09).

\section{Compliance with ethical standards}

Conflict of interest The authors declare that there is no conflict of interest.

Open Access This article is licensed under a Creative Commons Attribution 4.0 International License, which permits use, sharing, adaptation, distribution and reproduction in any medium or format, as long as you give appropriate credit to the original author(s) and the source, provide a link to the Creative Commons licence, and indicate if changes were made. The images or other third party material in this article are included in the article's Creative Commons licence, unless indicated otherwise in a credit line to the material. If material is not included in the article's Creative Commons licence and your intended use is not permitted by statutory regulation or exceeds the permitted use, you will need to obtain permission directly from the copyright holder. To view a copy of this licence, visit http://creativecommons.org/licenses/by/4.0/.

\section{References}

1. Alves, C.O., Nóbrega, A.B.: Nodal ground state solution to a biharmonic equation via dual method. J. Differ. Equ. 260, 5174-5201 (2016)

2. Ambrosio, V., Isernia, T., Rădulescu, V.D.: Concentration of positive solutions for a class of fractional p-Kirchhoff type equations. Proc. R. Soc. Edinb. Sect. A. (2020). https://doi.org/10.1017/prm.2020. 32

3. Ambrosio, V., Rădulescu, V.D.: Fractional double-phase patterns: concentration and multiplicity of solutions, J. Math. Pures Appl. (in press)

4. Arosio, A., Panizzi, S.: On the well-posedness of the Kirchhoff string. Trans. Am. Math. Soc. 348, 305-330 (1996)

5. Bartsch, T., Weth, T.: Three nodal solutions of singularly perturbed elliptic equations on domains without topology. Ann. Inst. H. Poincaré Anal. Non Linéaire 22, 259-281 (2005)

6. Bonheure, D., Santos, E., Ramos, M., Tavares, H.: Existence and symmetry of least energy nodal solutions for Hamiltonian elliptic systems. J. Math. Pures Appl. 104, 1075-1107 (2015)

7. Brändle, C., Colorado, E., Pablo, A., Sánchez, U.: A concave-convex elliptic problem involving the fractional Laplacian. Proc. R. Soc. Edinb. Sect. A 143, 39-71 (2013)

8. Caffarelli, L., Silvestre, L.: An extension problem related to the fractional Laplacian. Commun. Part. Differ. Equ. 32, 1245-1260 (2007)

9. Carrier, G.F.: On the nonlinear vibration problem of the elastic string. Q. Appl. Math. 3, 157-165 (1945)

10. Carrier, G.F.: A note on the vibrating string. Q. Appl. Math. 7, 97-101 (1949)

11. Chang, X.J., Wang, Z.Q.: Nodal and multiple solutions of nonlinear problems involving the fractional Laplacian. J. Differ. Equ. 256, 2965-2992 (2004)

12. Chang, X.J., Nie, Z., Wang, Z.Q.: Sign-changing solutions of fractional $p$-Laplacian problems. Adv. Nonlinear Stud. 19, 29-53 (2019)

13. D'Ancona, P., Shibata, Y.: On global solvability of nonlinear viscoelastic equations in the analytic category. Math. Methods Appl. Sci. 17, 477-489 (1994)

14. D'Ancona, P., Spagnolo, S.: Global solvability for the degenerate Kirchhoff equation with real analytic data. Invent. Math. 108, 247-262 (1992)

15. d'Avenia, P., Squassina, M., Zenari, M.: Fractional logarithmic Schrödinger equations. Math. Methods Appl. Sci. 38, 5207-5216 (2015)

16. Deng, Y.B., Shuai, W.: Sign-changing multi-bump solutions for Kirchhoff-type equations in $\mathbb{R}^{3}$. Discrete Contin. Dyn. Syst. Ser. A 38, 3139-3168 (2018) 
17. Figueiredo, G.M., Ikoma, N., Santos Júnior, J.R.: Existence and concentration result for the Kirchhoff type equations with general nonlinearities. Arch. Ration. Mech. Anal. 213, 931-979 (2014)

18. Figueiredo, G.M., Santos Júnior, J.R.: Existence of a least energy nodal solution for a SchrödingerKirchhoff equation with potential vanishing at infinity. J. Math. Phys. 56, 051506 (2015)

19. Fiscella, A., Valdinoci, E.: A critical Kirchhoff type problem involving a nonlocal operator. Nonlinear Anal. 94, 156-170 (2014)

20. Fiscella, A., Pucci, P., Zhang, B.L.: $p$-fractional Hardy-Schrödinger-Kirchhoff systems with critical nonlinearities. Adv. Nonlinear Anal. 8, 1111-1131 (2019)

21. Ghergu, M., Rădulescu, V.D.: Nonlinear PDEs. In: Mathematical Models in Biology, Chemistry and Population Genetics. Springer Monographs in Mathematics. Springer, Heidelberg (2012)

22. He, X.M., Zou, W.M.: Ground states for nonlinear Kirchhoff equations with critical growth. Ann. Mat. Pura Appl. 193, 473-500 (2014)

23. Ji, C., Fang, F., Zhang, B.L.: A multiplicity result for asymptotically linear Kirchhoff equations. Adv. Nonlinear Anal. 8, 267-277 (2019)

24. Kirchhoff, G.: Mechanik. Teubner, Leipzig (1883)

25. Li, F.Y., Gao, C., Zhu, X.: Existence and concentration of sign-changing solutions to Kirchhoff-type system with Hartree-type nonlinearity. J. Math. Anal. Appl. 448, 60-80 (2017)

26. Liang, S., Shi, S.: Soliton solutions to Kirchhoff type problems involving the critical growth in $\mathbb{R}^{N}$. Nonlinear Anal. 81, 31-41 (2013)

27. Liang, S., Zhang, J.: Existence of solutions for Kirchhoff type problems with critical nonlinearity in $\mathbb{R}^{3}$. Nonlinear Anal. Real World Appl. 17, 126-136 (2014)

28. Liang, S., Repovš, D., Zhang, B.: On the fractional Schrödinger-Kirchhoff equations with electromagnetic fields and critical nonlinearity. Comput. Math. Appl. 75, 1778-1794 (2018)

29. Lions, J.-L.: On some questions in boundary value problems of mathematical physics. In: Contemporary Developments in Continuum Mechanics and Partial Differential Equations, North-Holland Mathematics Studies, vol. 30, pp. 284-346. North-Holland, Amsterdam (1978)

30. Liu, J.Q., Liu, X.Q., Wang, Z.Q.: Sign-changing solutions for coupled nonlinear Schrödinger equations with critical growth. J. Differ. Equ. 261, 7194-7236 (2016)

31. Lu, S.: Signed and sign-changing solutions for a Kirchhoff-type equation in bounded domains. J. Math. Anal. Appl. 432, 965-982 (2015)

32. Mao, A.M., Zhang, Z.T.: Sign-changing and multiple solutions of Kirchhoff type problems without the P.S. condition. Nonlinear Anal. 70, 1275-1287 (2009)

33. Miranda, C.: Un'osservazione su un teorema di Brouwer. Boll. Un. Mat. Ital. 3, 5-7 (1940)

34. Molica Bisci, G., Rădulescu, V.D.: Ground state solutions of scalar field fractional Schrödinger equations. Calc. Var. Part. Differ. Equ. 54(3), 2985-3008 (2015)

35. Molica Bisci, G., Rădulescu, V.D., Servadei, R.: Variational Methods for Nonlocal Fractional Problems, Encyclopedia of Mathematics and Its Applications, vol. 162. Cambridge University Press, Cambridge (2016)

36. Pucci, P., Xiang, M., Zhang, B.: Multiple solutions for nonhomogeneous Schrödinger-Kirchhoff type equations involving the fractional $p$-Laplacian in $\mathbb{R}^{N}$. Calc. Var. Part. Differ. Equ. 54, 2785-2806 (2015)

37. Pucci, P., Xiang, M., Zhang, B.: Existence and multiplicity of entire solutions for fractional $p$-Kirchhoff equations. Adv. Nonlinear Anal. 5, 27-55 (2016)

38. Shuai, W.: Sign-changing solutions for a class of Kirchhoff-type problem in bounded domains. J. Differ. Equ. 259, 1256-1274 (2015)

39. Sun, J., Li, L., Cencelj, M., Gabrovšek, B.: Infinitely many sign-changing solutions for Kirchhoff type problems in $\mathbb{R}^{3}$. Nonlinear Anal. 186, 33-54 (2018)

40. Tang, X.H., Cheng, B.: Ground state sign-changing solutions for Kirchhoff type problems in bounded domains. J. Differ. Equ. 261, 2384-2402 (2016)

41. Tang, X.H., Chen, S.: Ground state solutions of Nehari-Pohozaev type for Kirchhoff-type problems with general potentials. Calc. Var. Part. Differ. Equ. 56, 1-25 (2017)

42. Truong, L.X.: The Nehari manifold for fractional $p$-Laplacian equation with logarithmic nonlinearity on whole space. Comput. Math. Appl. 78, 3931-3940 (2019)

43. Wang, D.B.: Least energy sign-changing solutions of Kirchhoff-type equation with critical growth. J. Math. Phys. 61, 011501 (2020)

44. Wang, Z.P., Zhou, H.S.: Sign-changing solutions for the nonlinear Schrödinger Poisson system in $\mathbb{R}^{N}$. Calc. Var. Part. Differ. Equ. 52, 927-943 (2015) 
45. Weth, T.: Energy bounds for entire nodal solutions of autonomous superlinear equations. Calc. Var. Part. Differ. Equ. 27, 421-437 (2006)

46. Willem, M.: Minimax Theorems, Progress in Nonlinear Differential Equations and Their Applications, vol. 24. Birkhäuser Boston Inc, Boston (1996)

47. Xiang, M., Hu, D., Yang, D.: Least energy solutions for fractional Kirchhoff problems with logarithmic nonlinearity. Nonlinear Anal. (2020). https://doi.org/10.1016/j.na.2020.111899

48. Xiang, M.Q., Rădulescu, V.D., Zhang, B.L.: Fractional Kirchhoff problems with critical TrudingerMoser nonlinearity. Calc. Var. Part. Differ. Equ. 58, 57 (2019)

49. Xiang, M.Q., Zhang, B.L., Rădulescu, V.D.: Superlinear Schrödinger-Kirchhoff type problems involving the fractional p-Laplacian and critical exponent. Adv. Nonlinear Anal. 9, 690-709 (2020)

50. Zhang, Z.T., Perera, K.: Sign changing solutions of Kirchhoff type problems via invariant sets of descentow. J. Math. Anal. Appl. 317, 456-463 (2006)

51. Zhang, B.L., Rădulescu, V.D., Wang, L.: Existence results for Kirchhoff-type superlinear problems involving the fractional Laplacian. Proc. R. Soc. Edinb. Sect. A 149, 1061-1081 (2019)

Publisher's Note Springer Nature remains neutral with regard to jurisdictional claims in published maps and institutional affiliations.

\section{Affiliations}

\section{Sihua Liang ${ }^{1,2} \cdot$ Vicenţiu D. Rădulescu ${ }^{3,4}{ }_{(D}$}

Sihua Liang

liangsihua@163.com

1 College of Mathematics and Informatics, Fujian Normal University, Qishan Campus, Fuzhou 350108, China

2 College of Mathematics, Changchun Normal University, Changchun 130032, People’s Republic of China

3 Faculty of Applied Mathematics, AGH University of Science and Technology, 30-059 Kraków, Poland

4 Department of Mathematics, University of Craiova, 200585 Craiova, Romania 